THEP 98/8

University of Freiburg

April 1998

hep-th/9805057

\title{
The Nambu-Goto Theory of Closed Bosonic Strings Moving in 1+3-Dimensional Minkowski Space: The Quantum Algebra of Observables
}

\author{
K. Pohlmeyer \\ Fakultät für Physik der Universität Freiburg, Hermann-Herder-Str. 3, \\ D-79104 Freiburg, Germany
}

\begin{abstract}
A relevant part of the quantum algebra of observables for the closed bosonic strings moving in 1+3-dimensional Minkowski space is presented in the form of generating relations involving still one, as yet undetermined, real free parameter.
\end{abstract}




\section{Introduction}

The present communication is part of an ongoing effort to describe the observable features of the Nambu-Goto field theory of relativistic, linearly extended geometric objects, called strings, in purely algebraic terms [6].

Conventionally, the Nambu-Goto theory is treated as any field theory of point-like objects with phase space constraints: auxiliary fields are introduced in order to give the action a quadratic form, BRST techniques are used to handle the constraints, special coordinates are introduced in order to "solve" the equations of motion, to define new dynamical variables and to facilitate the transition from the classical theory to the quantum theory, etc. ( $c f$, for instance, Ref. [1]). It is well-known that in its Euclidean version the classical Nambu-Goto theory of closed strings coincides with the theory of extremal surfaces (in whatever ambient space) and the global features of such extremal surfaces are by no means a trivial issue.

In the approach advocated here the string is not resolved into its local pieces. For closed bosonic strings moving in $1+(d-1)$-dimensional Minkowski space - in the following I shall restrict myself to this case - the differential geometric information (typically within a finite region of time and space) about the trajectory surface of the string is encoded in a countable set of piecewise conserved data independent of any chosen coordinatization. These observable data are called invariant charges. Since they are well enough localized and flexibly localized in ambient time and space, the computation of their mutual Poisson brackets does not pose any difficulties nor does it produce any ambiguities. Moreover, the polynomial ring of invariant charges closes under Poisson bracket operation. Hence this ring forms a Poisson algebra. The next task is a presentation of this algebra. It would be overly naive to expect a presentation in terms of classical Lie algebras. Instead one is confronted with the typical situation in combinatorial algebra: a description of the pertinent algebra in terms of relations imposed on a freely generated algebra or, more technically speaking, a description in terms of a quotient of a freely generated algebra by an ideal, the latter being generated by the relations just mentioned [10]. The difficulty with this description lies in the lack of an explicit characterization of those elements of the freely generated algebra which are contained in the ideal, or even in the lack of control over the "dimension" of the ideal. Unfortunately, no alternative ways of presenting the Poisson algebra under consideration are available. (Obviously, for the matter of higher dimensional extended geometric objects, the situation is not going to improve!) However, once a sufficiently large and coherent class of generators for the (classical) ideal has been identified, the passage to a relevant part of the associative quantum algebra of observables is achieved by means of a deformation of the generators based upon correspondence and consistency considerations. It is the central concern of the following sections to demonstrate for strings moving in $1+3$-dimensional Minkowski space how this deformation works in detail and which classical preparations are required.

In the past, the efforts of my collaborators and myself aimed at a presentation of the algebra of observables for strings moving in $1+2$-dimensional Minkowski space. 
The reason for choosing these particular dimensions was the simple structure of the stabilizer group $S O(2)$ of the momentum rest frame for the string. A "slight" irregularity concerning the assignment of structural roles to the various generators of the algebra was the price for the technical simplifications. In the meantime another (possibly related) drawback made itself felt: the observed systematics of the generators for the aforementioned ideal suggests that the final generators to appear involve among a vast variety of terms eightfold Poisson brackets for the classical theory and eightfold commutators for the quantum theory, respectively. The handling and processing of such expressions is beyond the computational means of my collaborators and myself. By contrast, for strings moving in $1+3$-dimensional Minkowski space there are indications that the collection of generators will be complete at a much earlier stage. This makes it worthwhile to pass to $1+3$-dimensional ambient Minkowski space and to put up with the complications reflected in the employment of Clebsch-Gordan coefficients and $6 j$-symbols.

\section{Classical Preparations}

Consider the algebra formed by the generators of the infinitesimal observable symmetry transformations of the Nambu-Goto theory of closed bosonic strings moving in $1+3$-dimensional Minkowski space. The structure of this algebra is given in terms of the Poincaré algebra corresponding to rigid Lorentz transformations and translations of the string in the ambient Minkowski space and of the algebra of (internal) invariant charges $\mathcal{Z}_{\mu_{1} \ldots \mu_{N}}^{+}$, built from the left movers

$$
u_{\mu_{i}}^{+}(\tau, \sigma):=p_{\mu_{i}}(\tau, \sigma)+\frac{1}{2 \pi \alpha^{\prime}} \partial_{\sigma} x_{\mu_{i}}(\tau, \sigma)
$$

and $\mathcal{Z}_{\mu_{1} \ldots \mu_{N}}^{-}$, built from the right movers

$$
u_{\mu_{i}}^{-}(\tau, \sigma):=p_{\mu_{i}}(\tau, \sigma)-\frac{1}{2 \pi \alpha^{\prime}} \partial_{\sigma} x_{\mu_{i}}(\tau, \sigma)
$$

Here $x_{\mu_{i}}(\tau, \sigma)$ and $p_{\mu_{i}}(\tau, \sigma)$ are the canonical string variables as functions of a "timelike" parameter $\tau$ and a "spacelike" parameter $\sigma$. The symbol $\alpha^{\prime}$ denotes the inverse string tension. $\mu_{i} \in\{0, \ldots, 3\}, i=1, \ldots, N, N=1,2,3, \ldots$.

$$
\begin{gathered}
\mathcal{Z}_{\mu_{1} \ldots \mu_{N}}^{ \pm}:=\oint \mathrm{d} \sigma u_{\mu_{1}}^{ \pm}(\tau, \sigma) \mathcal{R}_{\mu_{2} \ldots \mu_{N}}^{ \pm}(\tau, \sigma), \\
\mathcal{R}_{\mu_{2} \ldots \mu_{N}}^{ \pm}(\tau, \sigma):=\int_{\sigma+\omega(\tau)>\sigma_{2}>\sigma_{3}>\ldots>\sigma_{N}>\sigma} \mathrm{d} \sigma_{2} \cdots \int \mathrm{d} \sigma_{N} \prod_{i=2}^{N} u_{\mu_{i}}^{ \pm}\left(\tau, \sigma_{i}\right) .
\end{gathered}
$$

In the last formula the symbol $\omega(\tau)$ stands for the period of the canonical string variables as functions of the (closed string) curve parameter $\sigma$. The invariant charges 
$\mathcal{Z}_{\mu_{1} \ldots \mu_{N}}^{ \pm}$are unaffected by translations and transform covariantly under Lorentz transformations in the ambient Minkowski space. They form a Poisson algebra. This algebra can be identified with the tensor product of two Poisson algebras - one formed by the invariant charges $\mathcal{Z}_{\mu_{1} \ldots \mu_{N}}^{+}$, the other forned by the invariant charges $\mathcal{Z}_{\mu_{1} \ldots \mu_{N}}^{-}-$ which "Poisson commute" with each other. As multiplicative algebras, the two subalgebras are isomorphic and are generated by so-called standard invariants [8]: the first subalgebra is generated by standard invariants $\mathcal{Z}_{\mu_{1} \ldots \mu_{N}}^{+(K)}$, the second by standard invariants $\mathcal{Z}_{\mu_{1} \ldots \mu_{N}}^{-(K)}$ for certain values of $K: 1 \leq K \leq \frac{N+1}{2}$, and of $\mu_{1} \ldots \mu_{N}$. As Lie algebras, with the Poisson bracket operation as the composition law, they differ by a global factor -1 for the structure constants. Thus it suffices to analyze just one of the two subalgebras.

For definiteness, choose the Poisson algebra of the invariant (internal) charges $\mathcal{Z}_{\mu_{1} \ldots \mu_{N}}^{+(K)}$. Take advantage of the fact that the generators of translations

$$
\mathcal{P}_{\mu}=\oint_{\tau \text { fixed }} \mathrm{d} \sigma u_{\mu}^{+}(\tau, \sigma)
$$

are central elements of this algebra by treating them as c-numbers $p_{\mu}$. Specialize to vectors $p_{\mu}$ on an arbitrary, yet fixed mass shell with mass $\mathfrak{m}>0$ and positive energy.

Exploit Lorentz invariance of the full algebra of observables and pass to the rest frame of $p_{\mu}$ :

$$
\mathcal{P}_{\mu}=\mathfrak{m} \delta_{\mu}^{0}
$$

Denote the algebra obtained in this way by $\mathfrak{h} . \mathfrak{h}$ is an algebra with a $*$-operation given by complex conjugation.

Within this algebra $\mathfrak{h}$ the infinitesimal generators of rotations (in the momentum rest frame) are given w.r.t. a Lorentzian basis by

$$
\tilde{\mathbf{J}}_{i}=\sum_{j=1}^{3} \sum_{k=1}^{3} \frac{-2 \pi \alpha^{\prime}}{4 \mathfrak{m}} \varepsilon_{i j k} \mathcal{Z}_{0 j k}^{+(2)}, \quad i=1,2,3 .
$$

After multiplication by the imaginary unit, they are to be inserted into the first argument of the Poisson bracket $\{,\}^{\mathrm{PB}}$. The $\tilde{\mathbf{J}}_{i}$ 's are the left mover parts of the components of the spin vector.

Using the angular momentum basis instead of the Lorentzian basis, the triplet

$$
\tilde{\mathcal{J}}_{1,-1}:=\frac{1}{\sqrt{2}} \tilde{\mathbf{J}}_{-}, \quad \tilde{\mathcal{J}}_{1,0}:=\tilde{\mathbf{J}}_{3}, \quad \tilde{\mathcal{J}}_{1,+1}:=\frac{-1}{\sqrt{2}} \tilde{\mathbf{J}}_{+}
$$

with

$$
\tilde{\mathbf{J}}_{ \pm}:=\left(\tilde{\mathbf{J}}_{1} \pm i \tilde{\mathbf{J}}_{2}\right)
$$

forms itself an irreducible tensor variable

$$
\tilde{\mathcal{J}}_{1}=\left\{\tilde{\mathcal{J}}_{1, m} \mid m=-1,0,+1\right\}
$$


Here, in analogy to the irreducible tensor operator, an irreducible tensor variable

$$
\mathcal{O}_{j}=\left\{\mathcal{O}_{j, m} \mid m=-j,-j+1, \ldots,+j-1,+j\right\}
$$

- built from the left movers and carrying (integer) spin index $j \geq 0$ - is so defined that it satisfies the Poisson bracket relations

$$
\left\{i \tilde{\mathbf{J}}_{3}, \mathcal{O}_{j, m}\right\}^{\mathrm{PB}}=m \mathcal{O}_{j, m}, \quad\left\{i \tilde{\mathbf{J}}_{ \pm}, \mathcal{O}_{j, m}\right\}^{\mathrm{PB}}=\sqrt{(j \pm m+1)(j \mp m)} \mathcal{O}_{j, m \pm 1} .
$$

$\tilde{\mathcal{J}}_{1}$ carries the dimension of an action. The values of its components are appropriately stated in terms of complex multiples of a unit given by a typical action A.

I replace the Poisson bracket $\{,\}^{\mathrm{PB}}$, behaving as far as dimensions are concerned like an inverse action, by the rescaled Poisson bracket

$$
\{,\}_{\mathrm{PB}}=2 \pi \alpha^{\prime}\{,\}^{\mathrm{PB}}
$$

behaving dimensionally like a mass to the power minus two. As long as there is no risk of confusion with the anticommutator or the set theoretic symbol, I shall drop the subscript PB and simply denote the rescaled Poisson bracket by the symbol $\{$,$\} .$

With respect to the rescaled Poisson bracket $\{$,$\} the infinitesimal generators of$ rotations in the momentum rest frame are given by

$$
\mathbf{J}_{i}=\frac{-1}{4 \mathfrak{m}} \varepsilon_{i j k} \mathcal{Z}_{0 j k}^{+(2)} \quad\left(:=\sum_{j=1}^{3} \sum_{k=1}^{3} \frac{-1}{4 \mathfrak{m}} \varepsilon_{i j k} \mathcal{Z}_{0 j k}^{+(2)}\right), \quad i=1,2,3 .
$$

With the corresponding irreducible tensor variable

$$
\mathcal{J}_{1}=\left(\mathcal{J}_{1,-1}, \mathcal{J}_{1,0}, \mathcal{J}_{1,+1}\right)
$$

the Poisson bracket relations are

$$
\begin{gathered}
0=i\left\{\mathcal{J}_{1,-1}, \mathcal{J}_{1,+1}\right\}-\mathcal{J}_{1,0}, \quad 0=i\left\{\mathcal{J}_{1,0}, \mathcal{J}_{1, \pm 1}\right\} \mp \mathcal{J}_{1, \pm 1} ; \\
0=i\left\{\mathcal{J}_{1,0}, \mathcal{O}_{j, m}\right\}-m \mathcal{O}_{j, m}, \quad 0=i\left\{\mathcal{J}_{1, \pm 1}, \mathcal{O}_{j, m}\right\} \pm \frac{1}{\sqrt{2}} \sqrt{(j \pm m+1)(j \mp m)} \mathcal{O}_{j, m \pm 1}
\end{gathered}
$$

The values of the dynamical quantities $\mathcal{J}_{1, m}$ are appropriately stated as complex multiples of the unit

$$
\left(\frac{\mathbf{A}}{2 \pi \alpha^{\prime}}\right)=\left(\frac{\mathbf{A}}{2 \pi \alpha^{\prime} \mathfrak{m}^{2}}\right) \times \mathfrak{m}^{2}
$$

$\left(\mathbf{A} / 2 \pi \alpha^{\prime} \mathfrak{m}^{2}\right)$ being dimensionless. Actually, for any given invariant charge $\mathcal{Z}_{\mu_{1} \ldots \mu_{N}}^{+(K)}$ the appropriate scale is

$$
\left(\frac{\mathbf{A}}{2 \pi \alpha^{\prime}}\right)^{N-K} \times \mathfrak{m}^{2 K-N}=\left(\frac{\mathbf{A}}{2 \pi \alpha^{\prime} \mathfrak{m}^{2}}\right)^{N-K} \times \mathfrak{m}^{N}
$$


The power of the typical action A enters into the "degree"

$$
l:=N-K-1
$$

assigned to the invariant charge $\mathcal{Z}_{\mu_{1} \ldots \mu_{N}}^{+(K)}$. Poisson bracket operation $\left\{\mathcal{Z}_{\lambda_{1} \ldots \lambda_{N_{1}}}^{+\left(K_{1}\right)}, \mathcal{Z}_{\mu_{1} \ldots \mu_{N_{2}}}^{+\left(K_{2}\right)}\right\}$

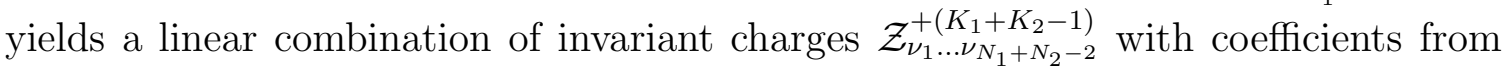
the real and complex numbers for a Lorentzian basis and an angular momentum basis, respectively. The degree $l$ behaves additively under Poisson bracket operation:

$$
\operatorname{degree}\left(\left\{\mathcal{Z}_{\lambda_{1} \ldots \lambda_{N_{1}}}^{+\left(K_{1}\right)}, \mathcal{Z}_{\mu_{1} \ldots \mu_{N_{2}}}^{+\left(K_{2}\right)}\right\}\right)=\operatorname{degree}\left(\mathcal{Z}_{\lambda_{1} \ldots \lambda_{N_{1}}}^{+\left(K_{1}\right)}\right)+\operatorname{degree}\left(\mathcal{Z}_{\mu_{1} \ldots \mu_{N_{2}}}^{+\left(K_{2}\right)}\right)
$$

in contrast to its behaviour under ordinary multiplication:

$$
\operatorname{degree}\left(\mathcal{Z}_{\lambda_{1} \ldots \lambda_{N_{1}}}^{+\left(K_{1}\right)} \cdot \mathcal{Z}_{\mu_{1} \ldots \mu_{N_{2}}}^{+\left(K_{2}\right)}\right)=\operatorname{degree}\left(\mathcal{Z}_{\lambda_{1} \ldots \lambda_{N_{1}}}^{+\left(K_{1}\right)}\right)+\operatorname{degree}\left(\mathcal{Z}_{\mu_{1} \ldots \mu_{N_{2}}}^{+\left(K_{2}\right)}\right)+1 \text {. }
$$

As it will turn out in the following section, this distinct behaviour is vital for the quantization, i.e. the passage from the Poisson algebra $\mathfrak{h}$ to the associative (noncommutative) algebra $\widehat{\mathfrak{h}}$.

Returning to the algebra of classical observables $\mathfrak{h}$, this very same degree $l$ endows $\mathfrak{h}$ with an $\mathbb{N}_{0}$-grading with respect to the Lie structure:

$$
\mathfrak{h}=\bigoplus_{l=0}^{\infty} \mathfrak{V}^{l}
$$

The homogeneous subspaces $\mathfrak{V}^{l}, l=0,1,2, \ldots$, are invariant under the $*$-operation. They are finite dimensional. For instance, the vector space $\mathfrak{V}^{0}$ is three-dimensional, the vector spaces $\mathfrak{V}^{1}, \mathfrak{V}^{2}$ and $\mathfrak{V}^{3}$ are 20-, 92- and 468-dimensional, respectively.

With respect to the second underlying structure of a Poisson algebra, the multiplication, $\mathfrak{h}$ is freely (not finitely) generated by the standard invariants: the number $n_{0}$ of generators contained in $\mathfrak{V}^{0}$ being three, the number $n_{l}$ of generators contained in each $\mathfrak{V}^{l}, l \geq 1$, being given by the formula

$$
n_{l}=n(4, l+2)-n(4, l+1)
$$

where

$$
n(4, N):=\frac{1}{N} \sum_{D \mid N} \mu(D) 4^{N / D}
$$

Here the sum extends over all divisors $D$ of $N$. The symbol $\mu(D)$ denotes the Möbius function of $D$ :

$$
\mu(D):=\left\{\begin{array}{cl}
1 & \text { if } D=1 \\
(-1)^{p} & \text { if } D \text { can be decomposed into exactly } p \text { different prime factors } \\
0 & \text { if some prime factors of } D \text { coincide. }
\end{array}\right.
$$


Consequently, the dimension of $\mathfrak{V}^{l}$ for $l \geq 1$ is given by a sum (over the number $q$ of generators occurring in the linearly independent monomials contained in $\mathfrak{V}^{l}$ ) of terms $t_{l}^{(q)}$

$$
\operatorname{dim}\left(\mathfrak{V}^{l}\right)=\sum_{q=1}^{l+1} t_{l}^{(q)}, \quad l \geq 1
$$

with

$$
t_{l}^{(q)}=\underbrace{\sum_{q_{1}=0}^{l} \sum_{q_{l}=0}^{l} \cdots \sum_{q_{1}}^{l}}_{\substack{1 \cdot q_{1}+2 \cdot q_{2}+\ldots+l \cdot q_{l}=l+1-q \\
q_{0}+\ldots+q_{l}=q}}\left(\begin{array}{c}
n_{0}+q_{0}-1 \\
q_{0}
\end{array}\right)\left(\begin{array}{c}
n_{1}+q_{1}-1 \\
q_{1}
\end{array}\right) \cdots\left(\begin{array}{c}
n_{l}+q_{l}-1 \\
q_{l}
\end{array}\right) .
$$

In this sense, the dimension of the vector spaces $\mathfrak{V}^{l}$ is under control.

The vector spaces $\mathfrak{V}^{l}$ can be decomposed into a direct sum of their positive parity and their negative parity parts:

$$
\mathfrak{V}^{l}=\mathfrak{V}_{+}^{l} \oplus \mathfrak{V}_{-}^{l}
$$

Actually, $\mathfrak{V}_{-}^{0}$ is the set $\{0\} \cdot \mathfrak{V}_{+}^{0}$ considered as a vector space coincides with the linear span of the invariant charges $\mathbf{J}_{i}, i=1,2,3$, or, equivalently, of the invariant charges $\mathcal{J}_{1, m}, m=-1,0,+1$.

Considered as an algebra w.r.t. Poisson bracket operation (Lie-Poisson algebra), $\mathfrak{V}_{+}^{0}$ coincides with the Lie algebra $s o(3)$ of the infinitesimal generators of rotations in the momentum rest frame

$$
\left\{i \mathbf{J}_{i}, \mathbf{J}_{j}\right\}=i \varepsilon_{i j k} \mathbf{J}_{k}
$$

Moreover, every vector space $\mathfrak{V}_{+}^{l}$ and $\mathfrak{V}_{-}^{l}$ is a representation space for the LiePoisson algebra $\mathfrak{V}_{+}^{0}$. Consequently, $\mathfrak{V}_{+}^{l}$ and $\mathfrak{V}_{-}^{l}$ can be decomposed into a direct sum of isotypical components corresponding to the spin $j: \mathfrak{V}_{j,+}^{l}$ and $\mathfrak{V}_{j,-}^{l}$, respectively. The index $j$ takes integer values between 0 and $l+1$.

Using this notation, for example $\mathfrak{V}_{+}^{0}, \mathfrak{V}_{+}^{1}$ and $\mathfrak{V}_{-}^{1}$ can be decomposed as

$$
\mathfrak{V}_{+}^{0}=\mathfrak{V}_{1,+}^{0}, \quad \mathfrak{V}_{+}^{1}=\mathfrak{V}_{0,+}^{1} \oplus \mathfrak{V}_{2,+}^{1}, \quad \mathfrak{V}_{-}^{1}=\mathfrak{V}_{1,-}^{1} \oplus \mathfrak{V}_{2,-}^{1}
$$

with pertinent dimensions $3,2 \oplus 10$ and $3 \oplus 5$, respectively.

The subspace $\mathfrak{V}_{0,+}^{1}$ is spanned by the elements

$$
\left(\mathcal{J}_{1}^{2}\right)_{0}:=\left\langle 0,0 \mid 1, m_{1} ; 1, m_{2}\right\rangle \mathcal{J}_{1, m_{1}} \mathcal{J}_{1, m_{2}} \quad\left(:=\sum_{m_{1}} \sum_{m_{2}}\left\langle 0,0 \mid 1, m_{1} ; 1, m_{2}\right\rangle \mathcal{J}_{1, m_{1}} \mathcal{J}_{1, m_{2}}\right)
$$

and

$$
\mathcal{B}_{0}^{(1)}:=\frac{1}{2} \sum_{j=1}^{3} \mathcal{Z}_{0 j 0 j}^{+(2)}
$$


the subspace $\mathfrak{V}_{2,+}^{1}$ by the components of the irreducible tensor variables

$$
\left(\mathcal{J}_{1}^{2}\right)_{2}=\left\{\left(\mathcal{J}_{1}^{2}\right)_{2, m} \mid m=-2, \ldots,+2\right\}
$$

and

$$
\mathcal{T}_{2}=\left\{\mathcal{T}_{2, m} \mid m=-2, \ldots,+2\right\}
$$

with

$$
\begin{aligned}
\left(\mathcal{J}_{1}^{2}\right)_{2, m} & :=\left\langle 2, m \mid 1, m_{1} ; 1, m_{2}\right\rangle \mathcal{J}_{1, m_{1}} \mathcal{J}_{1, m_{2}}, \\
\mathcal{T}_{2,-2} & :=\frac{-1}{8} \mathcal{Z}_{0101}^{+(2)}+\frac{1}{8} \mathcal{Z}_{0202}^{+(2)}+\frac{1}{4} \mathcal{Z}_{0102}^{+(2)}
\end{aligned}
$$

and $\mathcal{T}_{2, m}$ obtained by repeated action of $i \mathbf{J}_{+}$on $\mathcal{T}_{2,-2}$ according to formulae (四) and (2) , the subspace $\mathfrak{V}_{1,-}^{1}$ is spanned by the components of the irreducible tensor variable

$$
\mathcal{S}_{1}=\left\{\mathcal{S}_{1, m} \mid m=-1,0,+1\right\}
$$

with

$$
\mathcal{S}_{1,-1}:=\frac{1}{2 \sqrt{2}} \sum_{j=1}^{3}\left(\mathcal{Z}_{0 j 1 j}^{+(2)}-i \mathcal{Z}_{0 j 2 j}^{+(2)}\right)
$$

and $\mathcal{S}_{1, m}$ obtained by repeated action of $i \mathbf{J}_{+}$on $\mathcal{S}_{1,-1}$ according to formulae (11) and (2), and, finally, the subspace $\mathfrak{V}_{2,-}^{1}$ by the components of the irreducible tensor variable

$$
\mathcal{S}_{2}=\left\{\mathcal{S}_{2, m} \mid m=-2, \ldots,+2\right\}
$$

with

$$
\mathcal{S}_{2,-2}:=\frac{-i}{4} \mathcal{Z}_{0131}^{+(2)}+\frac{i}{4} \mathcal{Z}_{0232}^{+(2)}-\frac{1}{2} \mathcal{Z}_{0132}^{+(2)}
$$

and $\mathcal{S}_{2, m}$ obtained by repeated action of $i \mathbf{J}_{+}$on $\mathcal{S}_{2,-2}$ according to formulae (11) and (2).

The symbols $\left\langle j, m \mid j_{1}, m_{1} ; j_{2}, m_{2}\right\rangle$ above denote the Clebsch-Gordan coefficients of Condon and Shortley. The normalization of the irreducible tensor variables $\mathcal{O}_{j, m}$ is such that

$$
0=\mathcal{O}_{j, m}^{*}-(-1)^{m} \mathcal{O}_{j,-m}
$$

Nota bene: the tensor variables $\mathcal{J}_{1}, \mathcal{B}_{0}^{(1)}$ and $\mathcal{T}_{2}$ carry positive parity, whereas the tensor variables $\mathcal{S}_{1}$ and $\mathcal{S}_{2}$ carry negative parity.

Alternatively, the vector spaces $\mathfrak{V}_{+}^{l}$ and $\mathfrak{V}_{-}^{l}$ can be decomposed into direct sums of eigenspaces of $\left\{i \mathbf{J}_{3}, \cdot\right\}_{\mathrm{PB}}$ corresponding to eigenvalues $m \in\{-(l+1), \ldots,+(l+1)\}$

$$
\mathfrak{V}_{+}^{l}=\bigoplus_{m} \mathfrak{V}_{+, m}^{l}, \quad \mathfrak{V}_{-}^{l}=\bigoplus_{m} \mathfrak{V}_{-, m}^{l}
$$

This allows to compute successively the dimension of every subspace $\mathfrak{V}_{j,+}^{l}, 0 \leq j \leq$ $l+1$, as $(2 j+1)$ times the difference between the dimension of $\mathfrak{V}_{+, j}^{l}$ and the dimension 
of $\mathfrak{V}_{+, j+1}^{l}$, beginning with $j=l+1$ and $\operatorname{dim}\left(\mathfrak{V}_{+, l+2}^{l}\right)=0$. The same goes for the computation of the dimensions of the subspaces $\mathfrak{V}_{j,-}^{l}, 0 \leq j \leq l+1$. Note that the dimensions of $\mathfrak{V}_{+, 0}^{l}$ and $\mathfrak{V}_{-, 0}^{l}$ coincide with the number of irreducible spin multiplets in $\mathfrak{V}_{+}^{l}$ and $\mathfrak{V}_{-}^{l}$, respectively. By turns, in essentially the same manner as the dimension of $\mathfrak{V}^{l}$ was computed before, the dimensions of $\mathfrak{V}_{+, m}^{l}$ and $\mathfrak{V}_{-, m}^{l}$, $-(l+1) \leq m \leq+(l+1)$, can be computed from the numbers $n_{l^{\prime},+, m^{\prime}}$ and $n_{l^{\prime},-, m^{\prime}}$ of the generators of the symmetric algebra (i.e. the standard invariants) which are contained in the subspaces $\mathfrak{V}_{+, m^{\prime}}^{l^{\prime}}$ and $\mathfrak{V}_{-, m^{\prime}}^{l^{\prime}}, l^{\prime} \leq l,-\left(l^{\prime}+1\right) \leq m^{\prime} \leq+\left(l^{\prime}+1\right)$.

In their turn, these numbers can be obtained from the following formulae

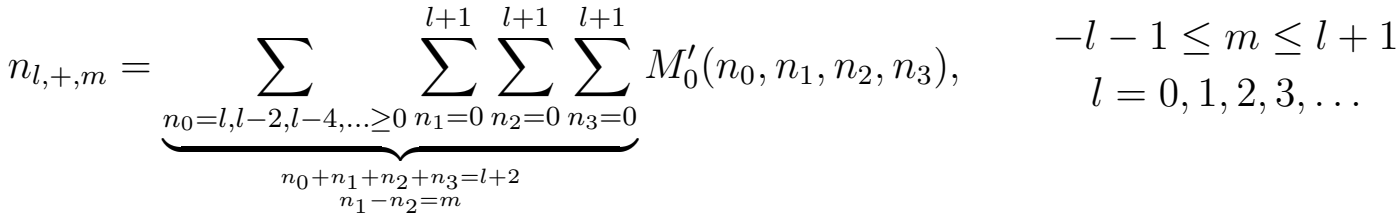

$$
\begin{aligned}
& n_{l,-, m}=\underbrace{\sum_{n_{1}-n_{2}=m}}_{n_{0}+n_{1}+n_{2}+n_{3}=l+2} \sum_{n_{0}=l-1, l-3, \ldots \geq 0} \sum_{n_{1}=0}^{l+1} \sum_{n_{2}=0}^{l+1} \sum_{n_{3}=0}^{l+1} M_{0}^{\prime}\left(n_{0}, n_{1}, n_{2}, n_{3}\right), \quad \begin{array}{c}
-l-1 \leq m \leq l+1 \\
l=1,2,3, \ldots
\end{array}
\end{aligned}
$$

with

$$
M_{0}^{\prime}\left(n_{0}, n_{1}, n_{2}, n_{3}\right)= \begin{cases}M_{0}\left(n_{0}, n_{1}, n_{2}, n_{3}\right)-M_{0}\left(n_{0}-1, n_{1}, n_{2}, n_{3}\right) & \text { for } n_{0} \geq 1 \\ M_{0}\left(0, n_{1}, n_{2}, n_{3}\right) & \text { for } n_{0}=0\end{cases}
$$

Here the symbol $M_{0}\left(k_{1}, k_{2}, \ldots, k_{r}\right), k_{1}, \ldots, k_{r} \in \mathbb{N}$, denotes the following function

$$
M_{0}\left(k_{1}, k_{2}, \ldots, k_{r}\right)=\frac{1}{K} \sum_{D \mid k_{\nu}, \nu=1,2, \ldots, r} \frac{\mu(D)(K / D) !}{\prod_{\nu=1}^{r}\left(k_{\nu} / D\right) !}, \quad K:=\sum_{\nu=1}^{r} k_{\nu} .
$$

To begin with the detailed structural analysis, I consider $\mathfrak{h}$ merely as an algebra with respect to multiplication, a freely generated one. I divide its generators (organized in irreducible tensor variables, in the sequel called irreducible tensor generators) into two disjoint sets:

i) the set of real, algebraically independent, positive parity, scalar generators $\mathcal{B}_{0}^{(l)}$, $l=1,3,5, \ldots$, of degree $l$, the linear span of which forms an abelian Lie algebra $\mathfrak{a}$, while the polynomials in $\mathcal{B}_{0}^{(l)}$ form a maximal abelian Lie subalgebra of the (Poisson) algebra $\mathfrak{h}$ [9], and

ii) a complementary set of suitably chosen irreducible tensor generators, each one of them carrying a well-defined degree $l \geq 0$. The polynomials of all these generators form a subalgebra $\mathfrak{U}$ of $\mathfrak{h}$ with respect to multiplication. 
Clearly, $\mathfrak{a} \cap \mathfrak{U}=\{0\}$ and, clearly, $\mathfrak{h}$ coincides with the polynomial ring in the elements of $\mathfrak{a}$ and $\mathfrak{U}$, the product in the algebra $\mathfrak{U}$ and the product in the polynomial ring being identified.

At this point, the Lie subalgebra $\mathfrak{a}$ is not uniquely defined, yet, in contrast to the maximal abelian Lie subalgebra of the (Poisson) algebra $\mathfrak{h}$, generated by it through multiplication. Also the subalgebra $\mathfrak{U}$ is not uniquely defined at this point. This non-uniqueness will be resolved shortly. In any case the subalgebra $\mathfrak{a}$ as a whole carries positive parity while $\mathfrak{U}$ contains elements of either parity:

$$
\begin{aligned}
\mathfrak{a} & =\mathfrak{a}_{+}, \\
\mathfrak{U} & =\mathfrak{U}_{+} \oplus \mathfrak{U}_{-} .
\end{aligned}
$$

The "grade" $p=l+1$ endows $\mathfrak{a}=\mathfrak{a}_{+}$and $\mathfrak{U}$ with an $\mathbb{N}$-gradation with respect to multiplication:

$$
\mathfrak{a}=\bigoplus_{p=2,4, \ldots} \mathfrak{a}_{+}^{(p)}, \quad \mathfrak{U}=\bigoplus_{p=1,2, \ldots} \mathfrak{U}^{(p)}=\bigoplus_{p=1,2, \ldots}\left(\mathfrak{U}_{+}^{(p)} \oplus \mathfrak{U}_{-}^{(p)}\right) .
$$

By means of this gradation, I define

$$
\begin{array}{ll}
\mathfrak{a}_{+}^{l}=\mathfrak{a}_{+}^{(l+1)} & \text { for } l=1,3,5, \ldots \\
\mathfrak{U}_{ \pm}^{l}=\mathfrak{U}_{ \pm}^{(l+1)} & \text { for } l=0,1,2, \ldots .
\end{array}
$$

Now, employing solely that part of the Lie structure of $\mathfrak{h}$ which is necessary to define the action of the generators $\mathbf{J}_{k}, k=1,2,3$, on the elements of $\mathfrak{h}$ via $\left\{i \mathbf{J}_{k}, \cdot\right\}_{\mathrm{PB}}$, I observe that each one of the subspaces $\mathfrak{a}_{+}^{l}, \mathfrak{U}_{+}^{l}$ and $\mathfrak{U}_{-}^{l}$ is a representation space for $s o(3) \cong \mathfrak{U}_{+}^{0}=\mathfrak{V}^{0}\left(\mathfrak{U}_{-}^{0}=\{0\}\right)$. Hence, each one of these subspaces can be decomposed into invariant subspaces corresponding to equivalent irreducible representations of so(3) labelled by the spin-value $j$ with $0 \leq j \leq l+1$, or, alternatively, into eigenspaces of $i \mathbf{J}_{3}$ corresponding to the eigenvalue $m$ with $-(l+1) \leq m \leq+(l+1)$. For the one-dimensional subspaces $\mathfrak{a}_{+}^{l}$ both decompositions are trivial, while for $\mathfrak{U}_{+}^{l}$ and $\mathfrak{U}_{-}^{l}$ they are highly non-trivial and helpful. As before, the $m$-decomposition provides the information about the dimension of the $j$-decomposition. Here are some examples:

$$
l=1: \quad \mathfrak{U}_{+}^{1}=\mathfrak{U}_{0,+}^{1} \oplus \mathfrak{U}_{2,+}^{1} \quad \text { and } \quad \mathfrak{U}_{-}^{1}=\mathfrak{U}_{1,-}^{1} \oplus \mathfrak{U}_{2,-}^{1}
$$

with respective dimensions $1 \oplus 10$ and $3 \oplus 5$;

$$
l=2: \quad \mathfrak{U}_{+}^{2}=\mathfrak{U}_{1,+}^{2} \oplus \mathfrak{U}_{2,+}^{2} \oplus \mathfrak{U}_{3,+}^{2} \quad \text { and } \quad \mathfrak{U}_{-}^{2}=\mathfrak{U}_{0,-}^{2} \oplus \ldots \oplus \mathfrak{U}_{3,-}^{2}
$$

with respective dimensions $15 \oplus 10 \oplus 21$ and $2 \oplus 12 \oplus 15 \oplus 14$;

$$
l=3: \quad \mathfrak{U}_{+}^{3}=\mathfrak{U}_{0,+}^{3} \oplus \ldots \oplus \mathfrak{U}_{4,+}^{3} \quad \text { and } \quad \mathfrak{U}_{-}^{3}=\mathfrak{U}_{0,-}^{3} \oplus \ldots \oplus \mathfrak{U}_{4,-}^{3}
$$

with respective dimensions $11 \oplus 30 \oplus 90 \oplus 42 \oplus 54$ and $6 \oplus 45 \oplus 70 \oplus 63 \oplus 36$. Actually, $\mathfrak{U}_{2,+}^{1} \equiv \mathfrak{V}_{2,+}^{1}, \mathfrak{U}_{1,-}^{1} \equiv \mathfrak{V}_{1,-}^{1}, \mathfrak{U}_{2,-}^{1} \equiv \mathfrak{V}_{2,-}^{1}$, while $\mathfrak{U}_{0,+}^{1}$ is the complex line $\left\{\lambda \cdot\left(\mathcal{J}_{1}^{2}\right)_{0} \mid \lambda \in\right.$ $\mathbb{C}\}$. 
Equally important as the knowledge of the dimensions of the various vector spaces $\mathfrak{U}_{j,+}^{l}$ and $\mathfrak{U}_{j,-}^{l}$, is the knowledge of the numbers $\bar{n}_{l, j,+}$ and $\bar{n}_{l, j,-}, 0 \leq j \leq l, l=$ $1,2, \ldots$, of the generators contained in $\mathfrak{U}_{j,+}^{l}$ and $\mathfrak{U}_{j,-}^{l}$, respectively, which generate the "multiplicative" algebra $\mathfrak{U}$ freely. This information is provided by the formulae $(l=1,2,3, \ldots)$

$$
\bar{n}_{l, j,-}:=(2 j+1)\left(n_{l,-, j}-n_{l,-, j+1}\right) \quad \text { for } j=0,1, \ldots, l+1
$$

with $n_{l,-, l+2}=0$, and

$$
\bar{n}_{l, j,+}:=(2 j+1) \times \begin{cases}\left(n_{l,+, j}-n_{l,+, j+1}\right) & \text { for } j=1, \ldots, l+1, \\ \left(n_{l,+, 0}-n_{l,+, 1}\right) & \text { for } j=0, l=0 \bmod 2, \\ \left(n_{l,+, 0}-n_{l,+, 1}-1\right) & \text { for } j=0, l=1 \bmod 2\end{cases}
$$

with $n_{l,+, l+2}=0$.

To proceed further I shall assume that the following conjecture holds true:

i) $\mathfrak{U}$ is a Poisson subalgebra of $\mathfrak{h}$. (In this case the $l$-decomposition $\mathfrak{U}=\bigoplus_{l=0}^{\infty} \mathfrak{U}^{l}=$ $\bigoplus_{l=0}^{\infty}\left(\mathfrak{U}_{+}^{l} \oplus \mathfrak{U}_{-}^{l}\right)$ corresponds to an $\mathbb{N}_{0}$-gradation of $\mathfrak{U}$ with respect to its Lie structure.)

ii) The derived algebra

$$
\left\{\bigoplus_{l=1}^{\infty} \mathfrak{U}^{l}, \bigoplus_{l=1}^{\infty} \mathfrak{U}^{l}\right\} \subset \bigoplus_{l=2}^{\infty} \mathfrak{U}^{l}
$$

contains all generators of $\mathfrak{U}$ with degree $l>1, \mathfrak{U}$ being considered here solely as an algebra with respect to multiplication.

iii) The generators $\mathcal{B}_{0}^{(l)}, l=1,3,5, \ldots$, which span the abelian subalgebra $\mathfrak{a} \subset \mathfrak{h}$, can be chosen such that the sum $\mathfrak{g}$ of $\mathfrak{a}$ and $\mathfrak{U}$ as Lie algebras is semi-direct, and such that they act as derivations on the Poisson algebra $\mathfrak{U}$

$$
\mathfrak{g}=\mathfrak{a} \ltimes \mathfrak{U} .
$$

This conjecture has been verified for $l \leq 7$, partly with the help of a computer program for symbolic computations.

Item $i i)$ of the conjecture implies that for $l \geq 2$ the $\bar{n}_{l, j,+}$ generators and the $\bar{n}_{l, j,-}$ generators of the multiplicative algebra $\mathfrak{U}$, which are contained in the subspaces $\mathfrak{U}_{j,+}^{l}$ and $\mathfrak{U}_{j,-}^{l}$, respectively, are obtainable in the form of algebraically independent iterated Poisson brackets with exactly $l$ entries from the vector spaces $\mathfrak{U}_{j,+}^{1}$ and $\mathfrak{U}_{j,-}^{1}$.

This is tantamount to saying that, as a Poisson algebra, $\mathfrak{U}$ is finitely generated by the irreducible tensors $\mathcal{J}_{1} \in \mathfrak{U}_{1,+}^{0}, \mathcal{T}_{2} \in \mathfrak{U}_{2,+}^{1}, \mathcal{S}_{1} \in \mathfrak{U}_{1,-}^{1}$ and $\mathcal{S}_{2} \in \mathfrak{U}_{2,-}^{1}$, or rather from two components of $\mathcal{J}_{1}$ and one component each of $\mathcal{T}_{2}, \mathcal{S}_{1}$ and $\mathcal{S}_{2}$. This resolves the previous non-uniqueness in the choice of $\mathfrak{U}$. 
Item $\mathrm{iii}$ ) of the conjecture resolves the previous non-uniqueness in the choice of $\mathfrak{a}$. $\mathfrak{h}$ is the symmetric algebra over $\mathfrak{g}$, the product in $\mathfrak{U}$ and the product in the symmetric algebra being identified.

For a presentation of the Poisson algebra $\mathfrak{h}$ it suffices to present the Poisson algebra $\mathfrak{U}$ and to specify the action of the basis elements of the abelian algebra $\mathfrak{a}$ on the generators of $\mathfrak{U}$.

$\mathfrak{U}$ will be presented as a two-fold quotient of the free Poisson algebra $\mathfrak{F}_{0}$ with abstract generators $\mathrm{J}_{1, m}, \mathrm{~T}_{2, m}, \mathrm{~S}_{1, m}$ and $\mathrm{S}_{2, m}$ carrying the same dimensions and degrees as in the Nambu-Goto theory. Besides, $\mathfrak{F}_{0}$ is equipped with a $*$-operation and a parity transformation $P$ which operate on the generators of $\mathfrak{F}_{0}$ as follows:

$$
\begin{array}{cl}
0=\left(\mathrm{J}_{1, m}\right)^{*}-(-1)^{m} \mathrm{~J}_{1,-m}, & 0=\left(\mathrm{T}_{2, m}\right)^{*}-(-1)^{m} \mathrm{~T}_{2,-m}, \\
0=\left(\mathrm{S}_{1, m}\right)^{*}-(-1)^{m} \mathrm{~S}_{1,-m}, & 0=\left(\mathrm{S}_{2, m}\right)^{*}-(-1)^{m} \mathrm{~S}_{2,-m}, \\
0=\left(\mathrm{J}_{1, m}\right)^{P}-\mathrm{J}_{1, m}, \quad 0=\left(\mathrm{T}_{2, m}\right)^{P}-\mathrm{T}_{2, m}, & 0=\left(\mathrm{S}_{1, m}\right)^{P}+\mathrm{S}_{1, m}, \quad 0=\left(\mathrm{S}_{2, m}\right)^{P}+\mathrm{S}_{2, m}
\end{array}
$$

and are extended to all of $\mathfrak{F}_{0}$ as a $*$-operation and an automorphism, respectively. Moreover $\mathfrak{F}_{0}$ is endowed with two gradings

a) with respect to the multiplicative structure given by the "grade" $(l+1)$;

b) with respect to the Lie structure given by the "degree" $l$.

The first quotient of $\mathfrak{F}_{0}$ is performed with respect to the graded ideal $\mathfrak{I}_{0}$. This quotient takes care of

i) the Poisson bracket relations of the components of $\mathcal{J}_{1}$ among each other,

ii) the Poisson bracket relations of the components of $\mathcal{J}_{1}$ with the components of $\mathcal{T}_{2}, \mathcal{S}_{1}$ and $\mathcal{S}_{2}$.

In other words, $\mathfrak{I}_{0}$ is generated by the following linear combinations: the right hand sides of the equations (II) and (2) (with the generators $\mathrm{J}_{1}$ and $\mathrm{T}_{2}, \mathrm{~S}_{1}, \mathrm{~S}_{2}$ substituted for the generators $\mathcal{J}_{1}$ and $\mathcal{O}_{j, m}$, respectively). All these generating elements are homogeneous in the degree $l$, in the spin value $j$, in the sign of the parity and in their reality properties. Hence the ideal $\mathfrak{I}_{0}$ is endowed with the same two-fold grading as $\mathfrak{F}_{0}$ and it is invariant under rotations in the momentum rest frame, under conjugation and parity transformation.

The result of the above identifications is the Poisson-*-algebra

$$
\mathfrak{F}=\mathfrak{F}_{0} / \mathfrak{I}_{0}
$$

with generators $J_{1}, T_{2}, S_{1}$ and $S_{2}$. $\mathfrak{F}$ is equipped with a two-fold grading and it is invariant under rotations in the momentum rest frame, under reflections and under conjugation, the latter operations being well-defined in $\mathfrak{F}$. The generators $J_{1}, T_{2}$, 
$S_{1}$ and $S_{2}$ carry the same dimensions and degrees as the respective variables in the Nambu-Goto theory.

The second and much more specific quotient of $\mathfrak{F}_{0}$ is taken with respect to an ideal $\mathfrak{I}$ generated by some particular polynomials in the generators $J_{1}, T_{2}, S_{1}$ and $S_{2}$ of $\mathfrak{F}$ and in their iterated Poisson brackets. This quotient takes care of the polynomial relations valid in the classical Nambu-Goto theory in the corresponding variables, polynomial relations other than those expressing the derivation property, antisymmetry property and Jacobi identity of the Poisson bracket and other than those expressing the covariance of the Poisson bracket and of the generators under rotations in the momentum rest frame, under reflections and under conjugation. This quotient must account for the difference of the dimensions of the (degree $l$, spin value $j$, parity plus or minus) subspaces $\mathfrak{F}_{j, \pm}^{l}$ of $\mathfrak{F}$ and the corresponding subspaces $\mathfrak{U}_{j, \pm}^{l}$ of $\mathfrak{U}$ in the classical Nambu-Goto theory:

The dimensions of the subspaces $\mathfrak{F}_{j, \pm}^{l}$ are computed essentially in the same manner as those of $\mathfrak{U}_{j, \pm}^{l}$, this time with the numbers $N_{ \pm, m}^{l}$ of generators (w.r.t. multiplication) contained in each subspace $\mathfrak{F}_{ \pm, m}^{l}, l \geq 1$ (corresponding to the $m$-decomposition of $\mathfrak{F}_{ \pm}^{l}$ ) obtained from the Witt formula applied to thirteen $l=1$ generators, viz. $T_{2, m}$, $S_{1, m}, S_{2, m}$ :

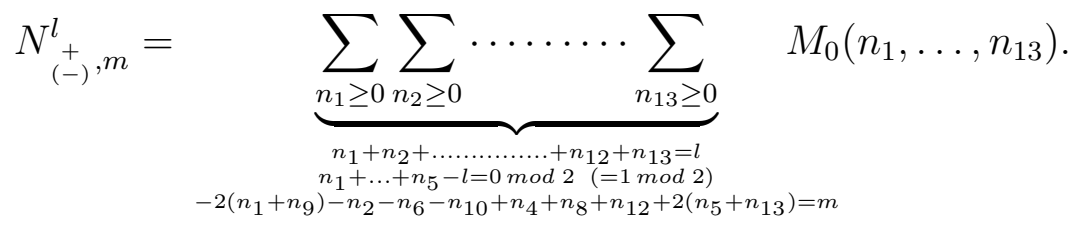

The concrete number and shape of the generators of the ideal $\mathfrak{I}$ is obtained from a basis of additional independent homogeneous polynomial relations in the variables $\mathcal{J}_{1, m}$, $\mathcal{T}_{2, m}, \mathcal{S}_{1, m}, \mathcal{S}_{2, m}$ and their iterated Poisson brackets, valid in the classical Nambu-Goto theory, without exploitation of the existence of the scalar elements $\mathcal{B}_{0}^{(l)}, l=1,3,5, \ldots$, their commutativity, and their specific actions on the generators $\mathcal{T}_{2, m}, \mathcal{S}_{1, m}$ and $\mathcal{S}_{2, m}$. Without loss of generality, these "UU-generating relations" can be arranged to be homogeneous in the degree $l$, in the spin value $j$, in the sign of the parity and in the behaviour under conjugation, and can be grouped into irreducible multiplets.

If, with the help of certain multiplets of polynomials $\mathcal{Q}_{j, \pm, r}^{l}\left(z_{1}, \ldots, z_{s}\right), r=$ $1,2,3, \ldots$, in the indeterminates $z_{1}, \ldots, z_{s}, s=1,2,3, \ldots$, these $\mathfrak{U}$-generating relations of the classical Nambu-Goto theory are cast into the form

$$
0=\mathcal{Q}_{j, \pm, r}^{l}\left(z_{1}, \ldots, z_{s}\right)
$$

for a definite identification of the indeterminates $z_{i}$ with the variables $\mathcal{J}_{1, m}, \ldots, \mathcal{S}_{2, m}$ and their (iterated) Poisson brackets, then the generators of $\mathfrak{I}$ are given by $\mathcal{Q}_{j, \pm, r}^{l}\left(z_{1}, \ldots, z_{s}\right)$ with the corresponding identification of the indeterminates $z_{i}$ with the generators $J_{1, m}, \ldots, S_{2, m}$ of $\mathfrak{F}$ and their (iterated) Poisson brackets.

The pertinent computations of the $\mathfrak{U}$-generating relations of the Nambu-Goto theory are performed with the help of the "modified Poisson brackets" for the building 
blocks $\mathcal{R}_{\mu_{1} \ldots \mu_{N}}^{t}$ of the invariant charges $\mathcal{Z}_{\mu_{1} \ldots \mu_{N}}^{+(K)}$ [8]. For invariant charges (expressed as polynomials in the building blocks $\mathcal{R}_{\mu_{1} \ldots \mu_{N}}^{t}$ ) the original Poisson bracket operation and the modified Poisson bracket operation give identical results. However, the use of the modified Poisson brackets offers considerable practical advantages over the use of the original Poisson brackets.

Obviously, there are no such relations for $l=0$ and $l=1$. The complete list of $\mathfrak{U}$-generating relations for $l=2,3$ and 4 is given below. For $l=4$ there are only 4 such relations and even these ones are not independent of the $l=2$ and $l=3$ relations. In fact, they are all induced by the Poisson bracket operation of the scalar element $\mathcal{B}_{0}^{(1)}$ from the $l=2$ and $l=3$ relations. Moreover, the emergence of "truly independent' generating relations for $l>5$ is rather unlikely, particularly in view of the growing assistance of more and more elements $\mathcal{B}_{0}^{(l)} \in \mathfrak{a}$ in the induction procedure via their Poisson bracket operation on the $\mathfrak{U}$-relations with lower degree and in view of the growing number of induced $\mathfrak{U}$-relations in the wake of the abelian commutation relations of more and more elements of $\mathcal{B}_{0}^{(l)} \in \mathfrak{a}$. In any case, the quotient $\mathfrak{U}=\mathfrak{F} / \mathfrak{I}$ still contains a multitude of non-trivial ideals w.r.t. either one of its underlying algebraic structures. Examples of such ideals are the set of all elements of $\mathfrak{U}$ carrying degree $l \geq k(k=1,2,3, \ldots)$ and the polynomials of (ordinary) degree $\geq k(k=2,3,4, \ldots)$ in the generators $\mathcal{J}_{1}, \mathcal{T}_{2}, \mathcal{S}_{1}, \mathcal{S}_{2}$ of $\mathfrak{U}$ and (iterated) Poisson brackets thereof.

In order to settle the issue of completeness of the generators of $\mathfrak{I}$ corresponding to a given set of $\mathfrak{U}$-generating relations, one might try to compare the dimensions of $\overline{\mathfrak{I}}_{j, \pm}^{l}$, the homogeneous subspaces of the ideal $\overline{\mathfrak{I}}$ with only these generators, $\overline{\mathfrak{I}} \subset \mathfrak{I}$, to the dimensions of $\mathfrak{F}_{j, \pm}^{l}$ and $\mathfrak{U}_{j, \pm}^{l}$. For the computation of the dimensions of $\overline{\mathfrak{I}}_{j, \pm}^{l}$, one could exploit the existing explicit Poisson bracket isomorphism between the graded ideal $\overline{\mathfrak{I}}$ in the graded Poisson algebra $\mathfrak{F}$ and a corresponding graded ideal $\overline{\mathfrak{i}}$ in the graded Lie algebra $\mathfrak{f}$

$$
\mathfrak{f}=\mathfrak{f}_{0} / \mathfrak{i}_{0}
$$

where $\mathfrak{f}_{0}$ denotes the free Lie algebra with abstract generators ${\stackrel{\circ}{J_{1, m}}}_{1}(l=0), \stackrel{\circ}{\mathrm{T}}_{2, m}$, $\stackrel{\circ}{S}_{1, m}, \stackrel{\circ}{S}_{2, m}(l=1)$ and $\mathfrak{i}_{0} \subset \mathfrak{f}_{0}$ the ideal defined in analogy to the ideal $\mathfrak{I}_{0} \subset \mathfrak{F}_{0}$. The generators of the ideal $\overline{\mathfrak{i}} \subset \mathfrak{f}$ are obtained from the generators of $\overline{\mathfrak{I}} \subset \mathfrak{F}$ by deleting all non-trivial products contributing to the latter (i.e. leaving only the linear combinations of the iterated Poisson brackets) and substituting the generators of $\mathfrak{f}$ for those of $\mathfrak{F}$. The resulting generators for $\overline{\mathfrak{i}}$ are much simpler than their counterparts for $\overline{\mathfrak{I}}$. So one might hope that it is easier to keep track of the dependencies among the elements of $\overline{\mathfrak{i}}$ than of the dependencies among the elements of $\overline{\mathfrak{I}}$. However, even in this simplified version, no practicable algorithm is known at present which would lead to an analytic counting formula for the relevant dimensions. Thus, comparison of the dimensions of $\overline{\mathfrak{I}}_{j, \pm}^{l}, \mathfrak{F}_{j, \pm}^{l}$ and $\mathfrak{U}_{j, \pm}^{l}$ is not a viable option for settling the issue of completeness of $\overline{\mathfrak{I}}$. At present, the only - albeit unsatisfactory - option seems to be a symbolic computer construction of the homogeneous subspaces of the quotient algebra $\mathfrak{f} / \overline{\mathfrak{i}}$ for values of $l$ ranging from 5 to 7 . Computer programs of this kind are 
available: see e.g. Ref. [3].

Here is the list of generating relations for the algebra $\mathfrak{U}$, formulated as nonidentically satisfied relations in the algebra $\mathfrak{F}$. The notation is explained at the end of the list.

\section{$\underline{l=2}$}

$$
\begin{aligned}
J^{P}=4^{-}: \quad 0= & \left\{T_{2}, S_{2}\right\}_{4} \\
J^{P}=3^{+}: \quad 0= & \left\{T_{2}, T_{2}\right\}_{3}+i\left\{S_{2}, S_{1}\right\}_{3}-i 16\left(J_{1}^{3}\right)_{3} ; \\
0= & \left\{S_{2}, S_{2}\right\}_{3}-i 2\left\{S_{2}, S_{1}\right\}_{3}+i 8\left(J_{1} \cdot T_{2}\right)_{3}+i 48\left(J_{1}^{3}\right)_{3} ; \\
J^{P}=3^{-}: \quad 0= & \left\{T_{2}, S_{2}\right\}_{3}-i\left\{T_{2}, S_{1}\right\}_{3}-i 8\left(J_{1} \cdot S_{2}\right)_{3} \\
J^{P}=2^{-}: \quad 0= & \left\{T_{2}, S_{2}\right\}_{2}+\frac{i}{3} \sqrt{\frac{7}{2}}\left\{T_{2}, S_{1}\right\}_{2}+i \frac{4}{3} \sqrt{14}\left(J_{1} \cdot S_{2}\right)_{2} ; \\
J^{P}=1^{+}: \quad 0= & \left\{S_{2}, S_{2}\right\}_{1}+i \sqrt{\frac{2}{3}}\left\{S_{2}, S_{1}\right\}_{1}+\frac{1}{6} \sqrt{5}\left\{S_{1}, S_{1}\right\}_{1} \\
& +i 16 \sqrt{\frac{2}{3}}\left(J_{1} \cdot T_{2}\right)_{1}+i 32 \sqrt{\frac{2}{15}}\left(J_{1} \cdot\left(J_{1}^{2}\right)_{0}\right)_{1} ;
\end{aligned}
$$

\section{$\underline{l=3}$}

$$
\begin{aligned}
& J^{P}=5^{-}: \\
& 0=\left\{\left\{S_{2}, S_{1}\right\}_{3}, S_{2}\right\}_{5} ; \\
& J^{P}= 4^{+}: \\
& 0=\left\{\left\{S_{2}, S_{1}\right\}_{2}, T_{2}\right\}_{4}+i \frac{4}{9} \sqrt{\frac{2}{3}}\left\{\left\{T_{2}, S_{1}\right\}_{3}, S_{1}\right\}_{4}+i \frac{40}{9} \sqrt{\frac{2}{3}}\left(J_{1} \cdot\left\{S_{2}, S_{1}\right\}_{3}\right)_{4} \\
&+i \frac{16}{3} \sqrt{\frac{2}{3}}\left(T_{2}^{2}\right)_{4}-i 4 \sqrt{\frac{2}{3}}\left(S_{2}^{2}\right)_{4}-i \frac{64}{3} \sqrt{\frac{2}{3}}\left(\left(J_{1}^{2}\right)_{2} \cdot T_{2}\right)_{4}-i \frac{128}{3} \sqrt{\frac{2}{3}}\left(\left(J_{1}^{2}\right)_{2}^{2}\right)_{4} ;
\end{aligned}
$$$$
J^{P}=4^{-} \text {: }
$$$$
0 \stackrel{(*)}{=}\left\{\left\{T_{2}, S_{1}\right\}_{2}, T_{2}\right\}_{4}+\frac{3}{2}\left\{\left\{S_{2}, S_{1}\right\}_{2}, S_{2}\right\}_{4}-i 10 \sqrt{\frac{2}{3}}\left(J_{1} \cdot\left\{T_{2}, S_{1}\right\}_{3}\right)_{4}
$$$$
+i 4 \sqrt{\frac{2}{3}}\left(T_{2} \cdot S_{2}\right)_{4}-i 8 \sqrt{\frac{2}{3}}\left(\left(J_{1}^{2}\right)_{2} \cdot S_{2}\right)_{4} ;
$$

$$
J^{P}=3^{+} \text {: }
$$

$$
\begin{aligned}
0 \stackrel{(*)}{=} & \left\{\left\{S_{2}, S_{1}\right\}_{2}, T_{2}\right\}_{3}+\frac{1}{9} \sqrt{10}\left\{\left\{S_{2}, S_{1}\right\}_{1}, T_{2}\right\}_{3}-i \frac{10}{27}\left\{\left\{T_{2}, S_{1}\right\}_{2}, S_{1}\right\}_{3} \\
& +i \frac{260}{81} \sqrt{2}\left(J_{1} \cdot\left\{S_{2}, S_{1}\right\}_{3}\right)_{3}-i \frac{40}{81}\left(J_{1} \cdot\left\{S_{2}, S_{1}\right\}_{2}\right)_{3}+\frac{212}{27} \sqrt{\frac{2}{3}}\left(S_{2} \cdot S_{1}\right)_{3} \\
& -i \frac{32}{9} \sqrt{\frac{2}{3}}\left(\left(J_{1}^{2}\right)_{2} \cdot T_{2}\right)_{3} ;
\end{aligned}
$$

$0 \stackrel{(*)}{=}\left\{\left\{T_{2}, S_{1}\right\}_{3}, S_{1}\right\}_{3}-\sqrt{2}\left\{\left\{T_{2}, S_{1}\right\}_{2}, S_{1}\right\}_{3}+\frac{26}{3}\left(J_{1} \cdot\left\{S_{2}, S_{1}\right\}_{3}\right)_{3}$

$$
+\frac{16}{3} \sqrt{2}\left(J_{1} \cdot\left\{S_{2}, S_{1}\right\}_{2}\right)_{3}+i 4 \sqrt{\frac{1}{3}}\left(S_{2} \cdot S_{1}\right)_{3}+48 \sqrt{3}\left(\left(J_{1}^{2}\right)_{2} \cdot T_{2}\right)_{3} ;
$$




$$
\begin{aligned}
& J^{P}=3^{-} \text {: } \\
& 0 \stackrel{(*)}{=} \quad\left\{\left\{T_{2}, S_{1}\right\}_{2}, T_{2}\right\}_{3}+\frac{1}{3} \sqrt{10}\left\{\left\{T_{2}, S_{1}\right\}_{1}, T_{2}\right\}_{3}-\frac{1}{6} \sqrt{\frac{5}{2}}\left\{\left\{S_{2}, S_{1}\right\}_{1}, S_{2}\right\}_{3} \\
& +i \frac{10}{9} \sqrt{2}\left\{\left\{S_{2}, S_{1}\right\}_{3}, S_{1}\right\}_{3}-i \frac{19}{36}\left\{\left\{S_{2}, S_{1}\right\}_{2}, S_{1}\right\}_{3}+i 2 \sqrt{2}\left(J_{1} \cdot\left\{T_{2}, S_{1}\right\}_{3}\right)_{3} \\
& -i \frac{4}{9}\left(J_{1} \cdot\left\{T_{2}, S_{1}\right\}_{2}\right)_{3}-i 28 \sqrt{\frac{2}{3}}\left(T_{2} \cdot S_{2}\right)_{3}+4 \sqrt{\frac{2}{3}}\left(T_{2} \cdot S_{1}\right)_{3} \\
& +i \frac{808}{9} \sqrt{\frac{2}{3}}\left(\left(J_{1}^{2}\right)_{2} \cdot S_{2}\right)_{3}+104 \sqrt{\frac{2}{3}}\left(\left(J_{1}^{2}\right)_{2} \cdot S_{1}\right)_{3} ; \\
& 0=\left\{\left\{S_{2}, S_{1}\right\}_{1}, S_{2}\right\}_{3}-i \frac{5}{3} \sqrt{5}\left\{\left\{S_{2}, S_{1}\right\}_{3}, S_{1}\right\}_{3}+i \frac{43}{3} \sqrt{\frac{1}{10}}\left\{\left\{S_{2}, S_{1}\right\}_{2}, S_{1}\right\}_{3} \\
& +i 24 \sqrt{\frac{1}{5}}\left(J_{1} \cdot\left\{T_{2}, S_{1}\right\}_{3}\right)_{3}+i \frac{8}{3} \sqrt{10}\left(J_{1} \cdot\left\{T_{2}, S_{1}\right\}_{2}\right)_{3}+i 16 \sqrt{\frac{3}{5}}\left(T_{2} \cdot S_{2}\right)_{3} \\
& -32 \sqrt{\frac{3}{5}}\left(T_{2} \cdot S_{1}\right)_{3}-i \frac{2528}{3} \sqrt{\frac{1}{15}}\left(\left(J_{1}^{2}\right)_{2} \cdot S_{2}\right)_{3}-192 \sqrt{\frac{3}{5}}\left(\left(J_{1}^{2}\right)_{2} \cdot S_{1}\right)_{3} ; \\
& J^{P}=2^{+} \text {: } \\
& 0=\left\{\left\{T_{2}, S_{1}\right\}_{1}, S_{2}\right\}_{2}-\frac{23}{20} \sqrt{\frac{1}{35}}\left\{\left\{S_{2}, S_{1}\right\}_{2}, T_{2}\right\}_{2}-\frac{41}{60}\left\{\left\{S_{2}, S_{1}\right\}_{1}, T_{2}\right\}_{2} \\
& -i \frac{61}{450} \sqrt{\frac{1}{14}}\left\{\left\{T_{2}, S_{1}\right\}_{3}, S_{1}\right\}_{2}+i \frac{287}{180} \sqrt{\frac{1}{10}}\left\{\left\{T_{2}, S_{1}\right\}_{2}, S_{1}\right\}_{2} \\
& +i \frac{13}{100} \sqrt{\frac{1}{6}}\left\{\left\{T_{2}, S_{1}\right\}_{1}, S_{1}\right\}_{2}-i \frac{232}{25} \sqrt{\frac{2}{7}}\left(J_{1} \cdot\left\{S_{2}, S_{1}\right\}_{3}\right)_{2} \\
& +i \frac{136}{15} \sqrt{\frac{2}{5}}\left(J_{1} \cdot\left\{S_{2}, S_{1}\right\}_{2}\right)_{2}-i \frac{51}{25} \sqrt{6}\left(J_{1} \cdot\left\{S_{2}, S_{1}\right\}_{1}\right)_{2} \\
& -\sqrt{\frac{1}{5}}\left(J_{1} \cdot\left\{S_{1}, S_{1}\right\}_{1}\right)_{2}+i \frac{436}{5} \sqrt{\frac{2}{105}}\left(T_{2}^{2}\right)_{2}+i \frac{171}{5} \sqrt{\frac{6}{35}}\left(S_{2}^{2}\right)_{2} \\
& +\sqrt{\frac{1}{15}}\left(S_{2} \cdot S_{1}\right)_{2}+i \frac{8824}{15} \sqrt{\frac{2}{105}}\left(\left(J_{1}^{2}\right)_{2} \cdot T_{2}\right)_{2} \\
& -i \frac{1232}{15} \sqrt{\frac{2}{15}}\left(\left(J_{1}^{2}\right)_{0} \cdot T_{2}\right)_{2}+i \frac{2304}{35} \sqrt{\frac{6}{5}}\left(\left(J_{1}^{2}\right)_{0} \cdot\left(J_{1}^{2}\right)_{2}\right)_{2} \text {; } \\
& 0=\left\{\left\{S_{2}, S_{1}\right\}_{3}, T_{2}\right\}_{2}-\sqrt{\frac{2}{5}}\left\{\left\{S_{2}, S_{1}\right\}_{2}, T_{2}\right\}_{2}+\frac{1}{3} \sqrt{14}\left\{\left\{S_{2}, S_{1}\right\}_{1}, T_{2}\right\}_{2} \\
& -i \frac{44}{45}\left\{\left\{T_{2}, S_{1}\right\}_{3}, S_{1}\right\}_{2}-\frac{i}{9} \sqrt{\frac{7}{5}}\left\{\left\{T_{2}, S_{1}\right\}_{2}, S_{1}\right\}_{2}+\frac{i}{5} \sqrt{\frac{7}{3}}\left\{\left\{T_{2}, S_{1}\right\}_{1}, S_{1}\right\}_{2} \\
& +i \frac{232}{45}\left(J_{1} \cdot\left\{S_{2}, S_{1}\right\}_{3}\right)_{2}-i \frac{32}{9} \sqrt{\frac{7}{5}}\left(J_{1} \cdot\left\{S_{2}, S_{1}\right\}_{2}\right)_{2}-i \frac{8}{5} \sqrt{\frac{7}{3}}\left(J_{1} \cdot\left\{S_{2}, S_{1}\right\}_{1}\right)_{2} \\
& -i 64 \sqrt{\frac{1}{15}}\left(T_{2}^{2}\right)_{2}-i 24 \sqrt{\frac{3}{5}}\left(S_{2}^{2}\right)_{2}+\frac{4}{3} \sqrt{\frac{70}{3}}\left(S_{2} \cdot S_{1}\right)_{2}-i \frac{1216}{3} \sqrt{\frac{1}{15}}\left(\left(J_{1}^{2}\right)_{2} \cdot T_{2}\right)_{2} \\
& +i \frac{128}{3} \sqrt{\frac{7}{15}}\left(\left(J_{1}^{2}\right)_{0} \cdot T_{2}\right)_{2}-i 256 \sqrt{\frac{3}{35}}\left(\left(J_{1}^{2}\right)_{0} \cdot\left(J_{1}^{2}\right)_{2}\right)_{2} \text {; } \\
& J^{P}=2^{-} \text {: } \\
& 0 \stackrel{(*)}{=}\left\{\left\{T_{2}, S_{2}\right\}_{1}, T_{2}\right\}_{2}+i \sqrt{\frac{10}{21}}\left\{\left\{T_{2}, S_{1}\right\}_{2}, T_{2}\right\}_{2}+i \sqrt{\frac{1}{6}}\left\{\left\{T_{2}, S_{1}\right\}_{1}, T_{2}\right\}_{2} \\
& +i \frac{5}{7} \sqrt{\frac{3}{2}}\left\{\left\{S_{2}, S_{1}\right\}_{1}, S_{2}\right\}_{2}+2 \sqrt{\frac{3}{7}}\left\{\left\{S_{2}, S_{1}\right\}_{3}, S_{1}\right\}_{2}-\frac{1}{2} \sqrt{\frac{5}{3}}\left\{\left\{S_{2}, S_{1}\right\}_{2}, S_{1}\right\}_{2} \\
& +\frac{1}{7}\left\{\left\{S_{2}, S_{1}\right\}_{1}, S_{1}\right\}_{2}-\frac{i}{14} \sqrt{\frac{5}{6}}\left\{\left\{S_{1}, S_{1}\right\}_{1}, S_{1}\right\}_{2}-i \frac{14}{5} \sqrt{\frac{2}{3}}\left(J_{1} \cdot\left\{T_{2}, S_{2}\right\}_{1}\right)_{2} \\
& +\frac{68}{5} \sqrt{\frac{3}{7}}\left(J_{1} \cdot\left\{T_{2}, S_{1}\right\}_{3}\right)_{2}-\frac{562}{21} \sqrt{\frac{1}{15}}\left(J_{1} \cdot\left\{T_{2}, S_{1}\right\}_{2}\right)_{2}-\frac{32}{7}\left(J_{1} \cdot\left\{T_{2}, S_{1}\right\}_{1}\right)_{2} \\
& -32 \sqrt{\frac{1}{35}}\left(T_{2} \cdot S_{2}\right)_{2}-i \frac{2}{7} \sqrt{10}\left(T_{2} \cdot S_{1}\right)_{2}-\frac{704}{45} \sqrt{\frac{1}{35}}\left(\left(J_{1}^{2}\right)_{2} \cdot S_{2}\right)_{2} \\
& +\frac{2176}{45} \sqrt{\frac{1}{5}}\left(\left(J_{1}^{2}\right)_{0} \cdot S_{2}\right)_{2}-i \frac{180}{7} \sqrt{10}\left(\left(J_{1}^{2}\right)_{2} \cdot S_{1}\right)_{2} \text {; }
\end{aligned}
$$




$$
\begin{aligned}
& 0=\left\{\left\{S_{2}, S_{1}\right\}_{1}, S_{2}\right\}_{2}-i \frac{11}{15} \sqrt{\frac{7}{2}}\left\{\left\{S_{2}, S_{1}\right\}_{3}, S_{1}\right\}_{2}+i \frac{7}{6} \sqrt{\frac{1}{10}}\left\{\left\{S_{2}, S_{1}\right\}_{2}, S_{1}\right\}_{2} \\
& +\frac{i}{10} \sqrt{\frac{3}{2}}\left\{\left\{S_{2}, S_{1}\right\}_{1}, S_{1}\right\}_{2}-\frac{6}{5}\left(J_{1} \cdot\left\{T_{2}, S_{2}\right\}_{1}\right)_{2}-\frac{i}{5} \sqrt{14}\left(J_{1} \cdot\left\{T_{2}, S_{1}\right\}_{3}\right)_{2} \\
& +i \frac{7}{3} \sqrt{\frac{2}{5}}\left(J_{1} \cdot\left\{T_{2}, S_{1}\right\}_{2}\right)_{2}+i \frac{8}{5} \sqrt{6}\left(J_{1} \cdot\left\{T_{2}, S_{1}\right\}_{1}\right)_{2}-i 2 \sqrt{\frac{42}{5}}\left(T_{2} \cdot S_{2}\right)_{2} \\
& +i \frac{344}{15} \sqrt{\frac{14}{15}}\left(\left(J_{1}^{2}\right)_{2} \cdot S_{2}\right)_{2}-i \frac{112}{15} \sqrt{\frac{2}{15}}\left(\left(J_{1}^{2}\right)_{0} \cdot S_{2}\right)_{2}-96 \sqrt{\frac{3}{5}}\left(\left(J_{1}^{2}\right)_{2} \cdot S_{1}\right)_{2} ; \\
& 0=\left\{\left\{S_{1}, S_{1}\right\}_{1}, S_{1}\right\}_{2}+48 \sqrt{\frac{1}{5}}\left(J_{1} \cdot\left\{T_{2}, S_{2}\right\}_{1}\right)_{2}+i 4 \sqrt{\frac{14}{5}}\left(J_{1} \cdot\left\{T_{2}, S_{1}\right\}_{3}\right)_{2} \\
& -i 4 \sqrt{2}\left(J_{1} \cdot\left\{T_{2}, S_{1}\right\}_{2}\right)_{2}+i 12 \sqrt{\frac{6}{5}}\left(J_{1} \cdot\left\{T_{2}, S_{1}\right\}_{1}\right)_{2}+8 \sqrt{3}\left(T_{2} \cdot S_{1}\right)_{2} \\
& -i \frac{64}{5} \sqrt{\frac{14}{3}}\left(\left(J_{1}^{2}\right)_{2} \cdot S_{2}\right)_{2}-i \frac{448}{5} \sqrt{\frac{2}{3}}\left(\left(J_{1}^{2}\right)_{0} \cdot S_{2}\right)_{2}-80 \sqrt{3}\left(\left(J_{1}^{2}\right)_{2} \cdot S_{1}\right)_{2} ; \\
& J^{P}=1^{+} \text {: } \\
& 0 \stackrel{(*)}{=}\left\{\left\{T_{2}, S_{1}\right\}_{1}, S_{2}\right\}_{1}-\frac{59}{12} \sqrt{\frac{1}{21}}\left\{\left\{S_{2}, S_{1}\right\}_{3}, T_{2}\right\}_{1}+\frac{41}{12} \sqrt{\frac{1}{15}}\left\{\left\{S_{2}, S_{1}\right\}_{2}, T_{2}\right\}_{1} \\
& -\frac{5}{12}\left\{\left\{S_{2}, S_{1}\right\}_{1}, T_{2}\right\}_{1}+i \frac{29}{12} \sqrt{\frac{1}{10}}\left\{\left\{T_{2}, S_{1}\right\}_{2}, S_{1}\right\}_{1}+i \frac{11}{12} \sqrt{\frac{1}{30}}\left\{\left\{T_{2}, S_{1}\right\}_{1}, S_{1}\right\}_{1} \\
& +\frac{14}{3} \sqrt{\frac{1}{5}}\left(J_{1} \cdot\left\{T_{2}, T_{2}\right\}_{1}\right)_{1}+\frac{i}{3} \sqrt{\frac{2}{5}}\left(J_{1} \cdot\left\{S_{2}, S_{1}\right\}_{2}\right)_{1}+i 2 \sqrt{\frac{6}{5}}\left(J_{1} \cdot\left\{S_{2}, S_{1}\right\}_{1}\right)_{1} \\
& -\frac{7}{3}\left(J_{1} \cdot\left\{S_{1}, S_{1}\right\}_{1}\right)_{1}+7 \sqrt{\frac{3}{5}}\left(S_{2} \cdot S_{1}\right)_{1}+i 28 \sqrt{\frac{2}{5}}\left(\left(J_{1}^{2}\right)_{2} \cdot T_{2}\right)_{1} ; \\
& 0=\left\{\left\{S_{2}, S_{1}\right\}_{3}, T_{2}\right\}_{1}-\frac{1}{2} \sqrt{35}\left\{\left\{S_{2}, S_{1}\right\}_{2}, T_{2}\right\}_{1}+\frac{1}{2} \sqrt{21}\left\{\left\{S_{2}, S_{1}\right\}_{1}, T_{2}\right\}_{1} \\
& -\frac{i}{2} \sqrt{\frac{105}{2}}\left\{\left\{T_{2}, S_{1}\right\}_{2}, S_{1}\right\}_{1}+\frac{i}{2} \sqrt{\frac{35}{2}}\left\{\left\{T_{2}, S_{1}\right\}_{1}, S_{1}\right\}_{1} \\
& -i 2 \sqrt{210}\left(J_{1} \cdot\left\{S_{2}, S_{1}\right\}_{2}\right)_{1}-6 \sqrt{35}\left(S_{2} \cdot S_{1}\right)_{1}-i 16 \sqrt{210}\left(\left(J_{1}^{2}\right)_{2} \cdot T_{2}\right)_{1} \text {; } \\
& J^{P}=1^{-} \text {: } \\
& 0=\left\{\left\{S_{2}, S_{1}\right\}_{1}, S_{2}\right\}_{1}-i \frac{11}{4} \sqrt{\frac{1}{10}}\left\{\left\{S_{2}, S_{1}\right\}_{2}, S_{1}\right\}_{1}+i \frac{5}{4} \sqrt{\frac{5}{6}}\left\{\left\{S_{2}, S_{1}\right\}_{1}, S_{1}\right\}_{1} \\
& +\frac{7}{24}\left\{\left\{S_{1}, S_{1}\right\}_{1}, S_{1}\right\}_{1}-3 \sqrt{\frac{1}{5}}\left(J_{1} \cdot\left\{T_{2}, S_{2}\right\}_{1}\right)_{1}-12 \sqrt{\frac{1}{5}}\left(J_{1} \cdot\left\{T_{2}, S_{2}\right\}_{0}\right)_{1} \\
& +i \frac{14}{3} \sqrt{10}\left(J_{1} \cdot\left\{T_{2}, S_{1}\right\}_{2}\right)_{1}-i 49 \sqrt{\frac{1}{30}}\left(J_{1} \cdot\left\{T_{2}, S_{1}\right\}_{1}\right)_{1}-i 3 \sqrt{\frac{2}{5}}\left(T_{2} \cdot S_{2}\right)_{1} \\
& +49 \sqrt{\frac{1}{15}}\left(T_{2} \cdot S_{1}\right)_{1}-i \frac{64}{3} \sqrt{10}\left(\left(J_{1}^{2}\right)_{2} \cdot S_{2}\right)_{1}+\frac{322}{3} \sqrt{\frac{1}{15}}\left(\left(J_{1}^{2}\right)_{2} \cdot S_{1}\right)_{1} \\
& -\frac{196}{3} \sqrt{\frac{1}{3}}\left(\left(J_{1}^{2}\right)_{0} \cdot S_{1}\right)_{1} \text {. }
\end{aligned}
$$

The $\mathfrak{U}$-generating relations marked by an asterisk $(*)$ result from induction of $l=2$ relations with the help of the scalar element $\mathcal{B}_{0}^{(1)} \in \mathfrak{a}$.

$$
\underline{l=4}
$$

$J^{P}=2^{-}: \quad$ one $\mathfrak{U}$-generating relation induced from the second of the $l=3$, $J^{P}=2^{-}$relations with the help of $\mathcal{B}_{0}^{(1)}$;

$J^{P}=1^{+}$: $\quad$ two $\mathfrak{U}$-generating relations induced from the two $l=3, J^{P}=1^{+}$ relations with the help of $\mathcal{B}_{0}^{(1)}$;

$J^{P}=1^{-}$: $\quad$ one $\mathfrak{U}$-generating relation induced from the only $l=3, J^{P}=1^{-}$ relation with the help of $\mathcal{B}_{0}^{(1)}$. 
Notation: The irreducible tensor variables $\left(A_{j_{1}} \cdot B_{j_{2}}\right)_{j_{3}}$ and $\left\{A_{j_{1}}, B_{j_{2}}\right\}_{j_{3}}$ are defined by

$$
\begin{aligned}
\left(A_{j_{1}} \cdot B_{j_{2}}\right)_{j_{3}} & :=\left\{\left(A_{j_{1}} \cdot B_{j_{2}}\right)_{j_{3}, m_{3}} \mid m_{3}=-j_{3}, \ldots,+j_{3}\right\} \\
\left\{A_{j_{1}}, B_{j_{2}}\right\}_{j_{3}} & :=\left\{\left\{A_{j_{1}}, B_{j_{2}}\right\}_{j_{3}, m_{3}} \mid m_{3}=-j_{3}, \ldots,+j_{3}\right\}
\end{aligned}
$$

with

$$
\begin{gathered}
\left(A_{j_{1}} \cdot B_{j_{2}}\right)_{j_{3}, m_{3}}:=\left\langle j_{3}, m_{3} \mid j_{1}, m_{1} ; j_{2}, m_{2}\right\rangle A_{j_{1}, m_{1}} \cdot B_{j_{2}, m_{2}}, \\
\left\{A_{j_{1}}, B_{j_{2}}\right\}_{j_{3}, m_{3}}:=\left\langle j_{3}, m_{3} \mid j_{1}, m_{1} ; j_{2}, m_{2}\right\rangle\left\{A_{j_{1}, m_{1}}, B_{j_{2}, m_{2}}\right\}_{\mathrm{PB}} .
\end{gathered}
$$

Notice the following symmetry properties:

$$
\begin{gathered}
\left(A_{j_{1}} \cdot B_{j_{2}}\right)_{j_{3}}=(-1)^{j_{1}+j_{2}-j_{3}}\left(B_{j_{2}} \cdot A_{j_{1}}\right)_{j_{3}}, \\
\left\{A_{j_{1}}, B_{j_{2}}\right\}_{j_{3}}=-(-1)^{j_{1}+j_{2}-j_{3}}\left\{B_{j_{2}}, A_{j_{1}}\right\}_{j_{3}} .
\end{gathered}
$$

Finally, turning to the action of the basis elements $\mathcal{B}_{0}^{(l)}, l=1,3,5, \ldots$, of the abelian Lie algebra $\mathfrak{a}$ on the generators $\mathcal{T}_{2}, \mathcal{S}_{1}, \mathcal{S}_{2}$ of $\mathfrak{U}$, the pertinent formulae are exact copies of the respective formulae valid in the classical Nambu-Goto theory. For $l=1$ and $l=3$, i.e. for

$$
\mathcal{B}_{0}^{(1)}:=\frac{1}{2} \sum_{j=1}^{3} \mathcal{Z}_{0 j 0 j}^{+(2)}
$$

and for

$$
\begin{aligned}
\mathcal{B}_{0}^{(3)} & :=12 \sum_{j=1}^{3} \mathcal{Z}_{000 j 000 j}^{+(4)}+i \frac{7}{2} \sqrt{\frac{3}{10}}\left\{\left\{\mathcal{T}_{2}, \mathcal{S}_{1}\right\}_{2}, \mathcal{S}_{2}\right\}_{0}+i \frac{15}{2} \sqrt{\frac{3}{10}}\left\{\left\{\mathcal{S}_{2}, \mathcal{S}_{1},\right\}_{2}, \mathcal{T}_{2}\right\}_{0} \\
& +\frac{21}{4 \sqrt{5}}\left\{\left\{\mathcal{T}_{2}, \mathcal{S}_{1},\right\}_{1}, \mathcal{S}_{1}\right\}_{0}+i 7 \sqrt{\frac{3}{10}}\left(\mathcal{J}_{1} \cdot\left\{\mathcal{T}_{2}, \mathcal{T}_{2}\right\}_{1}\right)_{0}-\frac{21}{2} \sqrt{5}\left(\mathcal{J}_{1} \cdot\left\{\mathcal{S}_{2}, \mathcal{S}_{1}\right\}_{1}\right)_{0} \\
& -\frac{28}{\sqrt{5}}\left(\mathcal{T}_{2}^{2}\right)_{0}-\frac{24}{\sqrt{5}}\left(\mathcal{S}_{2}^{2}\right)_{0}+8 \sqrt{3}\left(\mathcal{S}_{1}^{2}\right)_{0}-\frac{2}{3} \mathcal{B}_{0}^{(1)^{2}}+\frac{42}{\sqrt{5}}\left(\left(\mathcal{J}_{1}^{2}\right)_{2} \cdot \mathcal{T}_{2}\right)_{0},
\end{aligned}
$$

the latter formulae have been explicitly computed:

$$
\begin{aligned}
\left\{\mathcal{B}_{0}^{(1)}, \mathcal{T}_{2}\right\}_{2}= & i \sqrt{6}\left\{\mathcal{S}_{2}, \mathcal{S}_{1}\right\}_{2} \\
\left\{\mathcal{B}_{0}^{(1)}, \mathcal{S}_{1}\right\}_{1}= & -i 6 \sqrt{\frac{2}{5}}\left\{\mathcal{T}_{2}, \mathcal{S}_{2}\right\}_{1}+2 \sqrt{\frac{3}{5}}\left\{\mathcal{T}_{2}, \mathcal{S}_{1}\right\}_{1}-24 \sqrt{\frac{3}{5}}\left(\mathcal{J}_{1} \cdot \mathcal{S}_{2}\right)_{1} \\
& +i 12 \sqrt{2}\left(\mathcal{J}_{1} \cdot \mathcal{S}_{1}\right)_{1} ; \\
\left\{\mathcal{B}_{0}^{(1)}, \mathcal{S}_{2}\right\}_{2}= & -i 2 \sqrt{\frac{2}{3}}\left\{\mathcal{T}_{2}, \mathcal{S}_{1}\right\}_{2}-i 4 \sqrt{\frac{2}{3}}\left(\mathcal{J}_{1} \cdot \mathcal{S}_{2}\right)_{2}+12\left(\mathcal{J}_{1} \cdot \mathcal{S}_{1}\right)_{2} .
\end{aligned}
$$

The corresponding formulae for $\mathcal{B}_{0}^{(3)}$ are too lengthy, however, to be reproduced here. 
The $\mathfrak{U}$-generating relations formulated as non-identically satisfied relations in the algebra $\mathfrak{F}$ turn into identities when the generators of $\mathfrak{U}$ are substituted for the generators of $\mathfrak{F}$.

Remaining within the limitations of a conventional presentation, the classical action of the scalar element $\mathcal{B}_{0}^{\left(l^{\prime}\right)}$ of the abelian Lie algebra $\mathfrak{a}$ on the generators of $\mathfrak{U}$ is specified by the components of the result of the action w.r.t. a given basis of $\mathfrak{U}_{j, \pm}^{l^{\prime}+1}$. The choice of this basis is not canonical. The choice is mainly influenced by aspects of convenience.

On the other hand, the $\mathfrak{U}$-generating relations of degree $l$, spin $j$ and definite parity are quoted in the form of an expansion in terms of special basis elements of $\mathfrak{F}_{j, \pm}^{l}$. The latter ones are given as results of Poisson bracket and multiplication operations involving only subsets of selected basis elements of the subspaces $\mathfrak{F}_{j^{\prime}, \pm}^{l^{\prime}}$ : $l^{\prime}<l$. The said selection takes the $\mathfrak{U}$-generating relations of degree $l^{\prime}$, spin $j^{\prime}$ and parity plus or minus into account such that the subset of selected basis elements of each subspace $\mathfrak{F}_{j^{\prime}, \pm}^{l^{\prime}}, l^{\prime}<l$, turns into a basis of the respective subspace $\mathfrak{U}_{j^{\prime}, \pm}^{l^{\prime}}$ simply by substituting the generators of $\mathfrak{U}$ for the generators of $\mathfrak{F}$. In their turn, the $\mathfrak{U}$-generating relations together with the induced relations, all of them of degree $l$, spin $j$ and definite parity, also suggest the construction and subsequent selection of basis elements of $\mathfrak{F}_{j, \pm}^{l}$, the subset of selected basis elements virtually providing a basis of the respective subspace $\mathfrak{U}_{j,+}^{l}$ or $\mathfrak{U}_{j,-}^{l}$ : with the help of these relations express a maximal number of (as elements of $\mathfrak{F}$ ) linearly independent $l$-fold rescaled Poisson brackets, whose entries consist of generators of $\mathfrak{F}$, in the form of linear combinations of the remaining $l$-fold rescaled Poisson brackets and linearly independent products of selected basis elements of $\mathfrak{F}_{j^{\prime}, \pm}^{l^{\prime}}, l^{\prime}<l$. The said relations can always be solved for the $l$-fold Poisson brackets by virtue of the algebraic independence of the standard invariants 80 .

A complete set of basis elements of $\mathfrak{F}_{j,+}^{l}$ or $\mathfrak{F}_{j,-}^{l}$ is given first by a maximal set of linearly independent rescaled Poisson brackets whose entries consist of the generators of $\mathfrak{F}$ and, in addition, by a maximal set of linearly independent products of arbitrary basis elements of $\mathfrak{F}_{j^{\prime}, \pm}^{\prime}, l^{\prime}<l$, such that the following rules are satisfied: resulting degree $=l$, resulting spin $=j$ and resulting parity identical with the definite original parity. The selected subset of basis elements of $\mathfrak{F}_{j,+}^{l}$ or $\mathfrak{F}_{j,-}^{l}$ is obtained first by discarding all those $l$-fold rescaled Poisson brackets which have been expressed with the help of the generating and the induced relations in the form of the above mentioned linear combinations and, in addition, keeping only the linearly independent products of selected basis elements of $\mathfrak{F}_{j^{\prime}, \pm}^{l^{\prime}}, l^{\prime}<l$, satisfying the above rules for degree, spin and parity.

Note that in none of the above relations there is any explicit reference to the typical action $\mathbf{A}$, inverse string tension $\alpha^{\prime}$ or mass $\mathfrak{m}$. In fact, all relations are homogeneous in these parameters such that one can cast them into a form involving only dimensionless quantities and dimensionless operations. In the quantum theory this will be carried out explicitly. 


\section{Quantum Theory}

After the above classical preparations I now turn to the main objective of the present communication: the determination of the quantum algebra of observables. To facilitate the orientation I shall begin with some introductory remarks.

One conceivable way to tackle the problem under consideration is the following: first specify the exact quantum interpretation of the classical expressions for the generators $\mathcal{T}_{2}, \mathcal{S}_{1}, \mathcal{S}_{2}$ and $\mathcal{B}_{0}^{(l)}, l=1,3,5, \ldots$, and subsequently from the resulting commutation relations calculate the quantum algebra of observables. I shall not adopt this strategy in the sequel. In fact, I shall not construct any concrete non-trivial representation at all of the algebra in question. Instead, along the lines of Ref. [6], I postulate that the classical generators $\left(\mathcal{J}_{1},\right) \mathcal{T}_{2}, \mathcal{S}_{1}, \mathcal{S}_{2}$ and $\mathcal{B}_{0}^{(l)}, l=1,3,5, \ldots$, possess faithful quantum counterparts $\left(\widehat{\mathcal{J}}_{1},\right) \widehat{\mathcal{T}}_{2}, \widehat{\mathcal{S}}_{1}, \widehat{\mathcal{S}}_{2}$ and $\widehat{\mathcal{B}}_{0}^{(l)}, l=1,3,5, \ldots$, respectively, which carry exactly the same dimensions and have the same covariance properties under rotations, reflections and star operation as their classical partners into which they turn in the classical limit.

Further, I postulate that there is a one to one correspondence between the generating relations of the Poisson algebra with generators $\mathcal{J}_{1}, \mathcal{T}_{2}, \mathcal{S}_{1}, \mathcal{S}_{2}$ and $\mathcal{B}_{0}^{(l)}$, $l=1,3,5, \ldots$, and the generating relations of the associative non-commutative algebra with generators $\widehat{\mathcal{J}}_{1}, \widehat{\mathcal{T}}_{2}, \widehat{\mathcal{S}}_{1}, \widehat{\mathcal{S}}_{2}$ and $\widehat{\mathcal{B}}_{0}^{(l)}, l=1,3,5, \ldots$, and that there is a structural similarity (to be explained below) between the classical and the quantum generating relations. Consistency of these requirements - in particular under commutator operations - should remove remaining ambiguities.

Once a relevant part of the quantum algebra $\widehat{\mathfrak{g}}$ (or, equivalently, $\widehat{\mathfrak{h}}$ ) has been determined, one can indeed proceed to specify the exact quantum interpretation of the classical expressions for $\mathcal{T}_{2}, \mathcal{S}_{1}, \mathcal{S}_{2}$ and more and more scalar elements $\mathcal{B}_{0}^{(l)}$ by using the previously established relations and commutator actions as a guide line. Moreover, by invoking the technique of induced representations [5] one can finally arrive at a faithful representation of the Poincaré invariant version of the quantum algebra of observable symmetry transformations. However, this aspect of the quantization program has not been pursued in any detail, yet, and will not be pursued here. It will be the subject of future investigations.

Now I set about determining a relevant part of the quantum algebra of internal observables built from the left movers. From the very beginning I shall assume that the full quantum algebra of observables is Poincare invariant and that the components $\widehat{\mathcal{P}}_{\mu}$ of the energy momentum operator continue to be central elements of the algebra of internal invariant charges. As before, this assumption permits to treat the components $\widehat{\mathcal{P}}_{\mu}$ as concrete c-numbers $p_{\mu}$ and to pass to the rest frame of $p_{\mu}$ :

$$
\widehat{\mathcal{P}}_{\mu}=\mathfrak{m} \delta_{\mu}^{0} 11 .
$$

The associative non-commutative algebra of observables obtained in this way is denoted by $\widehat{\mathfrak{h}}$. It is postulated that $\widehat{\mathfrak{h}}$ is generated by elements $\widehat{\mathcal{J}}_{1}, \widehat{\mathcal{T}}_{2}, \widehat{\mathcal{S}}_{1}, \widehat{\mathcal{S}}_{2}$, and $\widehat{\mathcal{B}}_{0}^{(l)}$, 
$l=1,3,5, \ldots$. Further, since in the quantum theory the typical action $\mathbf{A}$ is given by $\hbar$ (= Planck's constant divided by $2 \pi$ ), it is postulated that the generators $\widehat{\mathcal{J}}_{1}$, $\widehat{\mathcal{T}}_{2}, \widehat{\mathcal{S}}_{1}, \widehat{\mathcal{S}}_{2}$ and $\widehat{\mathcal{B}}_{0}^{(l)}$ scale like dimensionless scale invariant operators $\widehat{\mathbb{Y}}_{1}, \widehat{\mathbb{X}}_{2}, \widehat{\mathcal{S}}_{1}, \widehat{\mathcal{S}}_{2}$ and $\widehat{\mathcal{B}}_{0}^{(l)}$ times $\left(\hbar / 2 \pi \alpha^{\prime} \mathfrak{m}^{2}\right) \mathfrak{m}^{2},\left(\hbar / 2 \pi \alpha^{\prime} \mathfrak{m}^{2}\right)^{2} \mathfrak{m}^{4},\left(\hbar / 2 \pi \alpha^{\prime} \mathfrak{m}^{2}\right)^{2} \mathfrak{m}^{4},\left(\hbar / 2 \pi \alpha^{\prime} \mathfrak{m}^{2}\right)^{2} \mathfrak{m}^{4}$ and $\left(\hbar / 2 \pi \alpha^{\prime} \mathfrak{m}^{2}\right)^{l+1} \mathfrak{m}^{2(l+1)}$, respectively. In particular, each one of these generators of the algebra $\widehat{\mathfrak{h}}$ - in the original as well as in the dimensionless form - is affiliated with a definite non-negative integer degree $l$ or - equivalently - with a definite positive order in Planck's constant. This order is given by the respective power in the dimensionless constant $\hbar /\left(2 \pi \alpha^{\prime} \mathfrak{m}^{2}\right)$. It agrees with the "grade" $(l+1)$ of the respective classical partner. To lowest order in Planck's constant, the Poisson bracket is replaced by the commutator with the help of the following substitution:

$$
\{,\}^{\mathrm{PB}} \rightarrow \frac{1}{i \hbar}[,]
$$

or, equivalently,

$$
\{,\}_{\mathrm{PB}} \longrightarrow \frac{1}{i\left(\frac{\hbar}{2 \pi \alpha^{\prime}}\right)}[,] .
$$

Requiring structural similarity with the Poisson algebra $\mathfrak{h}$, the associative algebra $\widehat{\mathfrak{h}}$ is postulated to be the enveloping algebra of $\widehat{\mathfrak{g}}:=\widehat{\mathfrak{a}} \ltimes \widehat{\mathfrak{U}}, \widehat{\mathfrak{U}}$ being an associative algebra. The product in the enveloping algebra and the product in $\widehat{\mathfrak{U}}$ are to be identified. The algebra $\widehat{\mathfrak{U}}$ is generated by $\widehat{\mathbb{X}}_{1}, \widehat{\mathbb{X}}_{2}, \widehat{\mathfrak{S}}_{1}, \widehat{\mathfrak{S}}_{2}$. The first summand $\widehat{\mathfrak{a}}$ is an abelian commutator Lie algebra generated by the scalar, (w.r.t. *-operation) self conjugate basis elements $\widehat{\mathcal{B}}_{0}^{(l)}, l=1,3,5, \ldots$ :

$$
\left[\widehat{\mathcal{B}}_{0}^{\left(l_{1}\right)}, \widehat{\mathcal{B}}_{0}^{\left(l_{2}\right)}\right]=0 \quad \text { for } l_{1}, l_{2} \in\{1,3,5, \ldots\},
$$

which act as derivations on $\widehat{\mathfrak{U}}$.

$\widehat{\mathfrak{U}}$ will be presented as a two-fold quotient of the free associative algebra $\widehat{\mathfrak{F}}_{0}$ with abstract generators $\widehat{J}_{1}, \widehat{T}_{2}, \widehat{S}_{1}, \widehat{S}_{2}$ of the same dimensions and affiliated with the same orders in Planck's constant as $\widehat{\mathcal{J}}_{1}, \widehat{\mathcal{T}}_{2}, \widehat{\mathcal{S}}_{1}, \widehat{\mathcal{S}}_{2}$, respectively. The order in $\hbar$ provides a grading of $\widehat{\mathfrak{F}}_{0}$ (w.r.t. multiplication).

In complete congruency with the classical situation, the first quotient of $\widehat{\mathfrak{F}}_{0}$ takes care of the commutation relations of the components of $\widehat{\mathcal{J}}_{1}$ among each other, of the commutation relations of the components of $\widehat{\mathcal{J}}_{1}$ with the components of $\widehat{\mathcal{T}}_{1}, \widehat{\mathcal{S}}_{1}$ and $\widehat{\mathcal{S}}_{2}$ and of the relations among each of the irreducible tensor operators under consideration resulting from the $*$-operation and parity transformation. The result of the above identifications is an associative algebra $\widehat{\mathfrak{F}}$ with generators $\widehat{J}_{1}, \widehat{T}_{2}, \widehat{S}_{1}, \widehat{S}_{2}$ of dimensions $\mathfrak{m}^{2}, \mathfrak{m}^{4}, \mathfrak{m}^{4}, \mathfrak{m}^{4}$ and of orders in Planck's constant 1, 2, 2, 2, respectively. The order in $\hbar$ provides an $\mathbb{N}$-grading of $\widehat{\mathfrak{F}}$ w.r.t. multiplication.

Given any concrete choice of the degree $l, l \in\{0,1,2, \ldots\}$ (or, equivalently, of the order $(l+1)$ in Planck's constant), of the spin $j, j \in\{0,1,2, \ldots, l+1\}$, and of the 
parity, translate the basis of the subspace $\mathfrak{F}_{j, \pm}^{l}$ of $\mathfrak{F}$ constructed before into a basis of the homogeneous subspace $\widehat{\mathfrak{F}}_{j, \pm}^{l}$ of $\widehat{\mathfrak{F}}$ according to the following rules:

i) replace each (simple) rescaled Poisson bracket $\{,\}_{j^{\prime}}:=2 \pi \alpha^{\prime}\{,\}_{j^{\prime}}^{\mathrm{PB}}$ of the classical irreducible tensor variables by $\left(i \hbar / 2 \pi \alpha^{\prime}\right)^{-1}$ times the commutator $[,]_{j^{\prime}}$ of their respective counterparts in $\widehat{\mathfrak{F}}$ without changing the original succession of brackets inside an iterated bracket;

ii) replace each (simple) product $(\cdot)_{j^{\prime}}$ of the classical irreducible tensor variables by $1 / 2$ times the anticommutator $\{,\}_{j^{\prime}}$ of their respective counterparts in $\widehat{\mathfrak{F}}$ without changing the original succession of brackets inside an iterated bracket.

(These rules are suggested by the relative simplicity of the recoupling formulae for (mixed) commutators and anticommutators of three irreducible tensor operators (see further below).)

Similarly, for every fixed degree $l$, spin $j$ and parity for which all classical relations - $\mathfrak{U}$-generating relations as well as induced relations - are explicitly known and for which the entire set of classical relations of lesser degree is also known in explicit form, translate the subset of selected basis elements of $\mathfrak{F}_{j, \pm}^{l}$ constructed before into a corresponding subset of selected basis elements of $\widehat{\mathfrak{F}}_{j, \pm}^{l}$. Finally, one more substitution $\left(\widehat{\mathbb{X}}_{1}, \widehat{X}_{2}, \widehat{\mathcal{S}}_{1}, \widehat{\mathcal{S}}_{2}\right.$ for $\widehat{J}_{1}, \widehat{T}_{2}, \widehat{S}_{1}, \widehat{S}_{2}$, respectively) turns the selected basis elements of $\widehat{\mathfrak{F}}_{j, \pm}^{l}$ into elements of the isotypical component $\widehat{\mathfrak{U}}_{j, \pm}^{l}$ of $\widehat{\mathfrak{U}}^{l} \subset \widehat{\mathfrak{U}}$. Under the provision that the consistency postulate is rigorously satisfied for all cycles of the deformation routine described below, the latter elements form a (linear) basis of the subspace $\widehat{\mathfrak{U}}_{j, \pm}^{l}$. The algebra $\widehat{\mathfrak{U}}$ is still the direct sum of all such vector spaces $\widehat{\mathfrak{U}}_{j, \pm}^{l}$ though this sum does not correspond any more to an $\mathbb{N}_{0}$-grading w.r.t. the commutator operation. Instead it corresponds to a filtration.

The second quotient of $\widehat{\mathfrak{F}}_{0}$, i.e. the first and only quotient of the graded algebra $\widehat{\mathfrak{F}}$, is performed w.r.t. an ideal $\widehat{\mathfrak{I}}$ whose generators are deduced from the (quantum) $\widehat{\mathfrak{U}}-$ generating relations. In their turn the $\widehat{\mathfrak{U}}$-generating relations are obtained, on the one hand, from the classical truly independent $\mathfrak{U}$-generating relations (see above) and the classical formulae for the respective actions of the basis elements $\mathcal{B}_{0}^{(l)}$ of the abelian algebra $\mathfrak{a}$ on the generators of $\mathfrak{U}$ by a fairly conservative deformation of these classical relations and formulae and, on the other hand, from the postulated commutativity of the quantum actions of different $\widehat{\mathcal{B}}_{0}^{(l)}$ 's. As a result of this deformation also the quantum action of the basis elements $\widehat{\mathcal{B}}_{0}^{(l)}$ of the abelian algebra $\widehat{\mathfrak{a}}$ on the generators of $\widehat{\mathfrak{U}}=\widehat{\mathfrak{F}} / \widehat{\mathfrak{I}}$ is given, albeit - to begin with - in parametrized form.

Now I shall describe the deformation procedure. Consider the $\mathfrak{U}$-generating relations without an asterisk, i.e. the truly independent generating relations of the classical Nambu-Goto theory. Since there are no classical $\mathfrak{U}$-generating relations at all for degree $l=0$ and $l=1$, and thus by the consistency postulate there will never be any 
quantum relations affiliated with these degrees, the corresponding subspaces $\widehat{\mathfrak{U}}_{j, \pm}^{l}$ of $\widehat{\mathfrak{U}}$ with $l=0$ and $l=1$ can be identified with the respective subspaces $\widehat{\mathfrak{F}}_{j, \pm}^{l}$ of $\widehat{\mathfrak{F}}$ :

$$
\widehat{\mathfrak{F}}_{1}^{0}=\widehat{\mathfrak{F}}_{1,+}^{0} ; \quad \widehat{\mathfrak{U}}_{1}^{0}=\widehat{\mathfrak{U}}_{1,+}^{0}
$$

where $\widehat{\mathfrak{F}}_{1,+}^{0}$ and $\widehat{\mathfrak{U}}_{1,+}^{0}$ are the linear spans of $\widehat{J}_{1, m}$ and $\widehat{\mathscr{V}}_{1, m}$, respectively;

$$
\widehat{\mathfrak{F}}_{+}^{1}=\widehat{\mathfrak{F}}_{2,+}^{1}, \quad \widehat{\mathfrak{F}}_{-}^{1}=\widehat{\mathfrak{F}}_{1,-}^{1} \oplus \widehat{\mathfrak{F}}_{2,-}^{1} ; \quad \widehat{\mathfrak{U}}_{+}^{1}=\widehat{\mathfrak{U}}_{2,+}^{1}, \quad \widehat{\mathfrak{U}}_{-}^{1}=\widehat{\mathfrak{U}}_{1,-}^{1} \oplus \widehat{\mathfrak{U}}_{2,-}^{1}
$$

where $\widehat{\mathfrak{F}}_{2,+}^{1}$ and $\widehat{\mathfrak{U}}_{2,+}^{1}$ are the linear spans of $\widehat{T}_{2, m}$ and $\widehat{X}_{2, m}$, respectively, and - with $i$ equal to 1 or $2-\widehat{\mathfrak{F}}_{i,-}^{1}$ and $\widehat{\mathfrak{U}}_{i,-}^{1}$ the linear spans of $\widehat{S}_{i, m}$ and $\widehat{\mathfrak{S}}_{i, m}$, respectively.

The first non-trivial cycle of the deformation routine begins with the deformation of the $\mathfrak{U}$-generating relations of lowest degree, i.e. of degree 2 , and the deformation of the formulae specifying the action of the scalar basis element $\mathcal{B}_{0}^{(1)}$ on the generators of $\mathfrak{U}$. Having completed the first non-trivial cycle of the deformation routine, advance degree by degree: from $l$ to $(l+1)$ as long as the consistency requirements are met and as far as the classical preparations permit.

A full cycle of the deformation routine, using the constructs of the previous cycles and/or the displayed features of the $l=0$ and $l=1$ subspaces, consists of the following successive steps (the first two of which are already familiar):

In every one of the truly independent $\mathfrak{U}$-generating relations of degree $(l+1)$

i) replace each (simple) rescaled Poisson bracket $\{,\}_{j}:=2 \pi \alpha^{\prime}\{,\}_{j}^{\mathrm{PB}}$ of the classical irreducible tensor variables by $\left(i \hbar / 2 \pi \alpha^{\prime}\right)^{-1}$ times the commutator $[,]_{j}$ of their respective counterparts in $\widehat{\mathfrak{F}}$ without changing the original succession of brackets inside an iterated bracket;

ii) replace each (simple) product $(\cdot)_{j}$ of the classical irreducible tensor variables by $1 / 2$ times the anticommutator $\{,\}_{j}$ of their respective quantum counterparts (without changing the original succession of brackets inside an iterated bracket);

iii) add the most general admissible quantum correction, i.e. the most general parametrized linear combination of the basis elements of $\widehat{\mathfrak{U}}_{j, \pm}^{l^{\prime}}$ with $0 \leq l^{\prime} \leq l$ compatible with the mass dimension, the order $(l+2)$ in Planck's constant, the spin, the parity and the reality property (under $*$-operation) of the initial classical relation.

$i v$ ) Divide the relation so obtained by $\left(\hbar / 2 \pi \alpha^{\prime}\right)^{l+2}$ to arrive at a dimensionless parametrized relation involving the dimensionless generators $\widehat{X}_{1}, \widehat{X}_{2}, \widehat{S}_{1}, \widehat{\mathbb{S}}_{2}$, dimensionless operations and dimensionless parameters.

At this point the deformed versions of a maximal set of linearly independent relations of degree $(l+1)$ are available, though partially in parametrized form only. The 
deformed versions of the complement of the truly independent $\mathfrak{U}$-generating relations have already been obtained (partially in parametrized form) in the course of the preceding cycle by way of induction employing among other things the possibly parametrized commutator action of scalar basis elements $\widehat{\mathcal{B}}_{0}^{\left(l^{\prime}\right)}$ with $l^{\prime}<l$. When all the parameters are finally fixed, each relation of the maximal set under discussion must turn into an identity in $\widehat{\mathfrak{U}}$.

After this digression, I shall resume the description of the successive steps making up a full cycle:

$v$ ) In case that $(l+1)$ is even, apply the above steps $i)-i i i)$ to the formulae specifying the classical action of the scalar basis element $\mathcal{B}_{0}^{(l)} \in \mathfrak{a}$ on the generators of $\mathfrak{U}$ to obtain the corresponding parametrized quantum action of the scalar basis element $\widehat{\mathcal{B}}_{0}^{(l)} \in \widehat{\mathfrak{a}}$ on the generators $\widehat{\mathbb{X}}_{2}, \widehat{\mathcal{S}}_{1}$ and $\widehat{\mathcal{S}}_{2}$ of $\widehat{\mathfrak{U}}$.

vi) Produce and analyze the set of all $\widehat{\mathfrak{U}}$-relations of degree $(l+2)$ induced, on the one hand, from the partially parametrized $\widehat{\mathfrak{U}}$-generating relations of degree $(l+1)$ and less in all possible, a priori independent ways including, in particular, the possibly parametrized commutator action of the basis elements of $\widehat{\mathfrak{a}}$ : $\widehat{\mathcal{B}}_{0}^{\left(l^{\prime}\right)}, l^{\prime}=1,3,5, \ldots, l^{\prime} \leq l$, and, on the other hand, from the postulated commutativity of the possibly parametrized commutator actions of different basis elements $\widehat{\mathcal{B}}_{0}^{\left(l^{\prime}\right)}$ and $\widehat{\mathcal{B}}_{0}^{\left(l^{\prime \prime}\right)}$ with $l^{\prime}+l^{\prime \prime} \leq l+1$ (cf the comments below).

Insisting on the absence of any quantum relation which does not correspond unambiguously to a valid classical relation - this is the decisive consistency postulate - derive as many independent restrictions as possible for the parameters involved and resolve these restrictions by assigning numerical values to some or all of the parameters and by expressing the remaining parameters with the help of as few (old or new) parameters as possible. The restrictions are algebraic. Without loss of generality the parameters both old and new may be chosen to be real.

vii) Deduce the respective generators (modulo parameter fixing) of $\widehat{\mathfrak{I}}$ from the possibly still parametrized $\widehat{\mathfrak{U}}$-generating relations of degree $(l+1)$.

viii) Start a new cycle of the deformation routine with the degree raised by one and determine - if possible - in the course of the due consistency checks, besides the restrictions for the additional parameters to be introduced, the numerical values of the parameters of the previous quantum relations and quantum actions or, at least, reduce the number of the free parameters involved in them.

Here is an example of the quantum deformation of a classical $\mathfrak{U}$-generating relation (without an asterisk) of degree $(l+1):(l+1)=2, J^{P}=1^{+}$, the classical relation is

$$
\begin{aligned}
0= & i\left\{S_{2}, S_{2}\right\}_{1}-\sqrt{\frac{2}{3}}\left\{S_{2}, S_{1}\right\}_{1}+\frac{i}{6} \sqrt{5}\left\{S_{1}, S_{1}\right\}_{1} \\
& -16 \sqrt{\frac{2}{3}}\left(J_{1} \cdot T_{2}\right)_{1}-32 \sqrt{\frac{2}{15}}\left(J_{1} \cdot\left(J_{1}^{2}\right)_{0}\right)_{1}
\end{aligned}
$$


its parametrized $\widehat{\mathfrak{U}}$-generating counterpart of order three in Planck's constant is given by

$$
\begin{aligned}
& 0=\left[\widehat{\mathscr{S}}_{2}, \widehat{\mathbb{S}}_{2}\right]_{1}+i \sqrt{\frac{2}{3}}\left[\widehat{\mathscr{S}}_{2}, \widehat{\mathbb{S}}_{1}\right]_{1}+\frac{1}{6} \sqrt{5}\left[\widehat{\mathscr{S}}_{1}, \widehat{\mathscr{S}}_{1}\right]_{1} \\
& -8 \sqrt{\frac{2}{3}}\left\{\widehat{X}_{1}, \widehat{X}_{2}\right\}_{1}-16 \sqrt{\frac{2}{15}}\left\{\widehat{X}_{1},\left(\widehat{X}_{1}^{2}\right)_{0}\right\}_{1}+f \sqrt{10} \widehat{X}_{1} \text {. }
\end{aligned}
$$

The corresponding parametrized quantum generator of $\widehat{\mathfrak{I}}$ is:

$$
\begin{aligned}
& {\left[\widehat{ঙ}_{2}, \widehat{\mathscr{S}}_{2}\right]_{1}+i \sqrt{\frac{2}{3}}\left[\widehat{\mathscr{S}}_{2}, \widehat{\mathbb{S}}_{1}\right]_{1}+\frac{1}{6} \sqrt{5}\left[\widehat{\mathscr{S}}_{1}, \widehat{\mathscr{S}}_{1}\right]_{1}} \\
& -8 \sqrt{\frac{2}{3}}\left\{\widehat{X}_{1}, \widehat{X}_{2}\right\}_{1}-16 \sqrt{\frac{2}{15}}\left\{\widehat{X}_{1},\left(\widehat{X}_{1}^{2}\right)_{0}\right\}_{1}+f \sqrt{10} \widehat{X}_{1} \text {. }
\end{aligned}
$$

The symbol $f$ employed above denotes a real valued parameter, which in its initial form, $F=\left(\frac{\hbar}{2 \pi \alpha^{\prime}}\right)^{2} f$, is of order two in Planck's constant ( $c f$ comments below).

An example of the quantum deformation of the classical formulae of degree $(l+1)$ describing the classical action of the basis element $\mathcal{B}_{0}^{(l)}$ of $\mathfrak{a}$ on $\mathfrak{U}$ is provided by $(l+1)=2, J^{P}=1^{-}$,

$$
\begin{aligned}
i\left\{\mathcal{B}_{0}^{(1)}, \mathcal{S}_{1}\right\}_{1}= & 6 \sqrt{\frac{2}{5}}\left\{\mathcal{T}_{2}, \mathcal{S}_{2}\right\}_{1}+i 2 \sqrt{\frac{3}{5}}\left\{\mathcal{T}_{2}, \mathcal{S}_{1}\right\}_{1}-i 24 \sqrt{\frac{3}{5}}\left(\mathcal{J}_{1} \cdot \mathcal{S}_{2}\right)_{1} \\
& -12 \sqrt{2}\left(\mathcal{J}_{1} \cdot \mathcal{S}_{1}\right)_{1}
\end{aligned}
$$

The quantum action of $\widehat{\mathcal{B}}_{0}^{(1)}$ on $\widehat{\mathcal{S}}_{1}$ is given by the parametrized relation of order three in Planck's constant

$$
\begin{aligned}
{\left[\widehat{\mathcal{S}}_{0}^{(1)}, \widehat{\mathcal{S}}_{1}\right]_{1}=} & -i 6 \sqrt{\frac{2}{5}}\left[\widehat{\mathbb{X}}_{2}, \widehat{\mathcal{S}}_{2}\right]_{1}+2 \sqrt{\frac{3}{5}}\left[\widehat{\mathbb{X}}_{2}, \widehat{\mathcal{S}}_{1}\right]_{1}-i 12 \sqrt{\frac{3}{5}}\left\{\widehat{\mathscr{X}}_{1}, \widehat{\mathcal{S}}_{2}\right\}_{1} \\
& -6 \sqrt{2}\left\{\widehat{\mathscr{X}}_{1}, \widehat{\mathcal{S}}_{1}\right\}_{1}+i d \widehat{\mathcal{S}}_{1} .
\end{aligned}
$$

Here, the symbol $d$ denotes a real valued parameter, which in its initial form, $D=$ $\frac{\hbar}{2 \pi \alpha^{\prime}} d$, is of order one in Planck's constant.

A few comments upon the deformation procedure may be helpful.

Concerning step iii): Each coefficient of the parametrized linear combination under discussion carries an explicit factor $\left(\hbar / 2 \pi \alpha^{\prime} \mathfrak{m}^{2}\right)^{l-l^{\prime}}, l^{\prime}<l$, and an explicit mass dimension in order to balance the difference between the order in Planck's constant and the mass dimension of the respective basis element of $\widehat{\mathfrak{U}}_{j, \pm}^{l^{\prime}}$ on the one hand and the common order in $\hbar$ and common mass dimension of the terms arising from the classical relation in question through operations $i$ ) and $i i$ ) on the other hand.

Concerning steps $i v$ ) - vi): It would be inconsistent with insertion of numerical values to assign to the parameters in question also in their dimensionless form definite respective degrees or - equivalently - orders in Planck's constant. Hence the individual terms of a parametrized or numerically specified $\widehat{\mathfrak{U}}$-generating relation in dimensionless form are no longer uniform w.r.t. the degree. The quantum counterparts of the respective terms of the classical relation still have the largest degree present in common, the smaller degrees being carried by components which arise from the individual 
constituents of the quantum correction.

Nevertheless, also in this form the degree $l$ or - equivalently - the order $(l+1)$ in Planck's constant of the relation before division can be read off and can be used as a label for the relation after division. The same goes for the dimensionless relations parametrizing the quantum action of the basis elements $\widehat{\mathcal{B}}_{0}^{\left(l^{\prime}\right)}$ of $\widehat{\mathfrak{a}}$ on $\widehat{\mathfrak{U}}$, for the remaining dimensionless $\widehat{\mathfrak{U}}$-generating relations induced with the help of the elements $\widehat{\mathcal{B}}_{0}^{\left(l^{\prime}\right)}$ and for the generators deduced from the dimensionless $\widehat{\mathfrak{U}}$-generating relations.

Of course, the degree $l$ no longer endows the associative algebras $\widehat{\mathfrak{F}}$ and $\widehat{\mathfrak{U}}$, and their respective subspaces $\widehat{\mathfrak{F}}_{j, \pm}$ and $\widehat{\mathfrak{U}}_{j, \pm}$, with an $\mathbb{N}_{0}$-grading w.r.t. the commutator operation. It endows them with a filtration and possibly - as will be discussed later - with a $\mathbb{Z}_{2}$-grading, instead. Moreover, in analogy to the classical situation, the degree behaves differently under (rescaled) commutator and under anticommutator operation. Consequently, no well-defined degree can be assigned to naive products of factors which do not commute with each other in the spin channel under consideration.

Concerning steps vi) - viii): Associativity of the algebra w.r.t. the underlying multiplication is frequently taken into account by arranging the entries $A_{j_{1}}, B_{j_{2}}, C_{j_{3}}$ of iterated commutators, anticommutators or mixtures thereof in a standard order with the help of the following three formulae. The schemes $\left\{\begin{array}{lll}j_{1} & j_{2} & j_{3} \\ l_{1} & l_{2} & l_{3}\end{array}\right\}$ denote the corresponding $6 j$-symbols 4 .

$$
\begin{aligned}
& {\left[\left[A_{j_{1}}, B_{j_{2}}\right]_{l}, C_{j_{3}}\right]_{L}=\sum_{k}(-1)^{k+j_{2}+j_{3}} \sqrt{(2 l+1)(2 k+1)} \times} \\
& \times\left((-1)^{l}\left\{\begin{array}{lll}
j_{2} & j_{1} & l \\
j_{3} & L & k
\end{array}\right\}\left[\left[A_{j_{1}}, C_{j_{3}}\right]_{k}, B_{j_{2}}\right]_{L}-\left\{\begin{array}{lll}
j_{1} & j_{2} & l \\
j_{3} & L & k
\end{array}\right\}\left[\left[B_{j_{2}}, C_{j_{3}}\right]_{k}, A_{j_{1}}\right]_{L}\right), \\
& {\left[\left\{A_{j_{1}}, B_{j_{2}}\right\}_{l}, C_{j_{3}}\right]_{L}=\sum_{k}(-1)^{k+j_{2}+j_{3}} \sqrt{(2 l+1)(2 k+1)} \times} \\
& \times\left((-1)^{l}\left\{\begin{array}{lll}
j_{2} & j_{1} & l \\
j_{3} & L & k
\end{array}\right\}\left\{\left[A_{j_{1}}, C_{j_{3}}\right]_{k}, B_{j_{2}}\right\}_{L}+\left\{\begin{array}{ccc}
j_{1} & j_{2} & l \\
j_{3} & L & k
\end{array}\right\}\left\{\left[B_{j_{2}}, C_{j_{3}}\right]_{k}, A_{j_{1}}\right\}_{L}\right), \\
& \left\{\left\{A_{j_{1}}, B_{j_{2}}\right\}_{l}, C_{j_{3}}\right\}_{L}=\sum_{k}(-1)^{k+j_{2}+j_{3}} \sqrt{(2 l+1)(2 k+1)} \times \\
& \times\left((-1)^{l+1}\left\{\begin{array}{lll}
j_{2} & j_{1} & l \\
j_{3} & L & k
\end{array}\right\}\left[\left[A_{j_{1}}, C_{j_{3}}\right]_{k}, B_{j_{2}}\right]_{L}+\left\{\begin{array}{lll}
j_{1} & j_{2} & l \\
j_{3} & L & k
\end{array}\right\}\left\{\left\{B_{j_{2}}, C_{j_{3}}\right\}_{k}, A_{j_{1}}\right\}_{L}\right) .
\end{aligned}
$$

There is a momentous difference between the first two formulae on the one hand and the third formula on the other hand: whereas all terms of the first two formulae involve a common number of commutator operations and a common number of anticommutator operations, the first term inside the parenthesis on the right hand side of the third formula employs two commutator operations instead of the two anticommutator operations of the remaining terms of this formula. Thus, in general, elements of the algebra $\widehat{\mathfrak{h}}$ in their dimensionless form, homogeneous w.r.t. the order 
in Planck's constant, split off elements of lesser degree when they are subjected to manipulations involving the third formula. This does not happen when they are subjected to manipulations involving either one of the first two formulae.

Of course, there exist also valid classical versions of the above three formulae. They are obtained by substituting Poisson brackets for the commutator brackets in the first two formulae and by dropping the first term inside the parenthesis on the right hand side of the third formula. In fact, these classical versions have been used over and over again beforehand in the course of the classical considerations.

Concerning step vi): Suppose that one has confined oneself to the subspace $\left(\bigoplus_{l=1}^{k-1} \mathfrak{a}^{l}\right)$ $\oplus\left(\bigoplus_{l=0}^{k} \mathfrak{F}^{l}\right)$ of $\mathfrak{h}$ for any fixed $k \geq 1$ and that one has determined the set of all classical relations and actions available under this confinement. Then the consistency of the Poisson algebra $\mathfrak{h}$ guarantees that the relations, obtained from the above set by all possible moves of induction into the subspace $\bigoplus_{l=0}^{k+1} \mathfrak{F}^{l}$, never lash back at the subspace $\left(\bigoplus_{l=1}^{k-1} \mathfrak{a}^{l}\right) \oplus\left(\bigoplus_{l=0}^{k} \mathfrak{F}^{l}\right)$ by imposing (algebraic) relations for the elements of the latter which do not turn into identities when the initial relations and actions are taken into account.

In the quantum theory based on the deformation of the Poisson algebra, the consistency of the latter guarantees the following much weaker property of the corresponding set of partially parametrized deformed relations and actions: in case that induction of these relations and actions produces degree $(k+1)$-relations involving as their only respective contribution with degree $(k+1)$ a linear combination of anticommutators, then each such linear combination in its entirety turns into a sum of terms with lesser degree when the initial deformed relations and actions are taken into account without further adjustment of their parameters.

The consistency postulate requires that the resulting relations with degree $\leq k$ turn into identities when appropriate numerical values are assigned once and for all to some of the parameters or to all of them and when the initial deformed relations and actions are taken into consideration with these numerical values for their respective parameters. There is no escape from an overdetermined system of equations for the parameters that would not violate the consistency postulate.

So far the deformation routine has been carried out for the cycles of degree two and three:

In the course of the cycle of degree two, the first non-trivial cycle, six free real parameters were introduced: in their initial form five of first order and one of second order in Planck's constant. Step vi) of the deformation routine did not yield any restriction on these parameters.

In the course of the cycle of degree three, twenty-nine additional free real parameters were introduced: twenty one initially of first, seven initially of second and one initially of third order in Planck's constant. After a lot of processing, step vi) of the deformation routine furnished a system of linear and quadratic restrictions for the 
thirty-five parameters. The system received multiple contributions from each of the final spin-parity channels $J^{P}=0^{ \pm}, 2^{ \pm}, 3^{ \pm}$and $4^{ \pm}$. At first sight the system looked hopelessly overdetermined. From it without much difficulty a system of twenty-six independent homogeneous linear equations for twenty-six parameters was split off, all of the latter in their initial form of first order in Planck's constant. Necessarily these twenty-six parameters had to be set equal to zero. These insertions immediately implied that also the parameter initially of third order in Planck's constant had to be set equal to zero. At this point the original system of linear and quadratic equations was reduced to a system of linear inhomogeneous equations for the remaining eight parameters, all of which initially of second order in Planck's constant. (Multiple) contributions to this system came from the final spin-parity channels $J^{P}=0^{-}, 2^{ \pm}$and $3^{ \pm}$. Without prior knowledge this system still looked grossly overdetermined. At the end of the day, however, it turned out that the latter system was not overdetermined at all. On the contrary, it furnished only seven independent linear inhomogeneous equations for the remaining eight parameters. The numerical value of one of these parameters appearing in the basic parametrization of the quantum deformation was fixed as a rational number, to wit $16 / 5$. The rest of the equations was used to express six of the other seven parameters in terms of the only parameter initially of second order in Planck's constant which made its appearance already in the course of the cycle of degree two. The coefficients of the respective expressions are rational numbers.

Here are the basic data of the quantum algebra of observables of the NambuGoto theory as they have been obtained so far. They are quoted in condensed form in terms of those "fundamental" generators of $\widehat{\mathfrak{I}}$ corresponding to truly independent $\widehat{\mathfrak{U}}$-generating relations and of the quantum action of $\widehat{\mathcal{B}}_{0}^{(1)}$ on $\widehat{\mathfrak{U}}$. They still require the fixing and subsequent substitution of the numerical value of the residual parameter $f$.

$\underline{\text { Fundamental generators of } \widehat{\mathfrak{I}} \text { of degree two: }}$

$$
\begin{aligned}
& J^{P}=4^{-}: \quad\left[\widehat{\mathbb{K}}_{2}, \widehat{\mathbb{S}}_{2}\right]_{4} ; \\
& J^{P}=3^{+}: \quad\left[\widehat{\mathbb{X}}_{2}, \widehat{\mathbb{X}}_{2}\right]_{3}+i\left[\widehat{\mathscr{S}}_{2}, \widehat{S}_{1}\right]_{3}+16\left(\widehat{X}_{1}^{3}\right)_{3} ; \\
& {\left[\widehat{\mathscr{S}}_{2}, \widehat{\mathscr{S}}_{2}\right]_{3}-i 2\left[\widehat{\mathscr{S}}_{2}, \widehat{\mathscr{S}}_{1}\right]_{3}-4\left\{\widehat{\mathbb{X}}_{1}, \widehat{\mathbb{X}}_{2}\right\}_{3}-48\left(\widehat{X}_{1}^{3}\right)_{3} \text {; }} \\
& J^{P}=3^{-}: \quad\left[\widehat{\mathbb{X}}_{2}, \widehat{\mathbb{S}}_{2}\right]_{3}-i\left[\widehat{\mathbb{X}}_{2}, \widehat{\mathscr{S}}_{1}\right]_{3}+4\left\{\widehat{\mathbb{X}}_{1}, \widehat{\mathscr{S}}_{2}\right\}_{3} \text {; } \\
& J^{P}=2^{-}: \quad\left[\widehat{\mathbb{X}}_{2}, \widehat{\mathbb{S}}_{2}\right]_{2}+\frac{i}{3} \sqrt{\frac{7}{2}}\left[\widehat{\mathbb{X}}_{2}, \widehat{S}_{1}\right]_{2}-\frac{2}{3} \sqrt{14}\left\{\widehat{\mathbb{X}}_{1}, \widehat{S}_{2}\right\}_{2} ; \\
& J^{P}=1^{+}: \quad\left[\widehat{\mathbb{S}}_{2}, \widehat{\mathbb{S}}_{2}\right]_{1}+i \sqrt{\frac{2}{3}}\left[\widehat{\mathbb{S}}_{2}, \widehat{\mathbb{S}}_{1}\right]_{1}+\frac{1}{6} \sqrt{5}\left[\widehat{\mathbb{S}}_{1}, \widehat{\mathbb{S}}_{1}\right]_{1} \\
& -8 \sqrt{\frac{2}{3}}\left\{\widehat{X}_{1}, \widehat{X}_{2}\right\}_{1}-16 \sqrt{\frac{2}{15}}\left\{\widehat{X}_{1},\left(\widehat{X}_{1}^{2}\right)_{0}\right\}_{1}+f \sqrt{10} \widehat{X}_{1}
\end{aligned}
$$


$\underline{\text { Fundamental generators of } \widehat{\mathfrak{I}} \text { of degree three: }}$

$$
\begin{aligned}
& J^{P}=5^{-}: \\
& {\left[\left[\widehat{S}_{2}, \widehat{S}_{1}\right]_{3}, \widehat{S}_{2}\right]_{5}} \\
& J^{P}=4^{+} \text {: } \\
& {\left[\left[\widehat{\mathbb{S}}_{2}, \widehat{\mathscr{S}}_{1}\right]_{2}, \widehat{\mathbb{X}}_{2}\right]_{4}+i \frac{4}{9} \sqrt{\frac{2}{3}}\left[\left[\widehat{\mathbb{X}}_{2}, \widehat{\mathscr{S}}_{1}\right]_{3}, \widehat{\mathscr{S}}_{1}\right]_{4}-\frac{20}{9} \sqrt{\frac{2}{3}}\left\{\widehat{X}_{1},\left[\widehat{\mathscr{S}}_{2}, \widehat{\mathscr{S}}_{1}\right]_{3}\right\}_{4}-i \frac{16}{3} \sqrt{\frac{2}{3}}\left(\widehat{\mathbb{X}}_{2}^{2}\right)_{4}} \\
& +i 4 \sqrt{\frac{2}{3}}\left(\widehat{\mathscr{S}}_{2}^{2}\right)_{4}+i \frac{32}{3} \sqrt{\frac{2}{3}}\left\{\left(\widehat{X}_{1}^{2}\right)_{2}, \widehat{\mathbb{X}}_{2}\right\}_{4}+i \frac{128}{3} \sqrt{\frac{2}{3}}\left(\left(\widehat{X}_{1}^{2}\right)_{2}^{2}\right)_{4} ; \\
& J^{P}=3^{-} \text {: } \\
& {\left[\left[\widehat{\mathscr{S}}_{2}, \widehat{S}_{1}\right]_{1}, \widehat{S}_{2}\right]_{3}-i \frac{5}{3} \sqrt{5}\left[\left[\widehat{\mathscr{S}}_{2}, \widehat{\mathscr{S}}_{1}\right]_{3}, \widehat{S}_{1}\right]_{3}+i \frac{43}{3} \sqrt{\frac{1}{10}}\left[\left[\widehat{\mathscr{S}}_{2}, \widehat{\mathscr{S}}_{1}\right]_{2}, \widehat{S}_{1}\right]_{3}} \\
& -12 \sqrt{\frac{1}{5}}\left\{\widehat{\mathbb{X}}_{1},\left[\widehat{\mathbb{X}}_{2}, \widehat{\mathscr{S}}_{1}\right]_{3}\right\}_{3}-\frac{4}{3} \sqrt{10}\left\{\widehat{\mathbb{X}}_{1},\left[\widehat{\mathbb{K}}_{2}, \widehat{\mathscr{S}}_{1}\right]_{2}\right\}_{3}-i 8 \sqrt{\frac{3}{5}}\left\{\widehat{\mathbb{X}}_{2}, \widehat{\mathscr{S}}_{2}\right\}_{3} \\
& +16 \sqrt{\frac{3}{5}}\left\{\widehat{\mathbb{X}}_{2}, \widehat{\mathscr{S}}_{1}\right\}_{3}+i \frac{1264}{3} \sqrt{\frac{1}{15}}\left\{\left(\widehat{X}_{1}^{2}\right)_{2}, \widehat{\mathscr{S}}_{2}\right\}_{3}+96 \sqrt{\frac{3}{5}}\left\{\left(\widehat{X}_{1}^{2}\right)_{2}, \widehat{\mathscr{S}}_{1}\right\}_{3} ; \\
& J^{P}=2^{+} \text {: } \\
& {\left[\left[\widehat{\mathbb{X}}_{2}, \widehat{\mathscr{S}}_{1}\right]_{1}, \widehat{\mathscr{S}}_{2}\right]_{2}-\frac{23}{20} \sqrt{\frac{1}{35}}\left[\left[\widehat{\mathscr{S}}_{2}, \widehat{\mathbb{S}}_{1}\right]_{2}, \widehat{\mathbb{X}}_{2}\right]_{2}-\frac{41}{60}\left[\left[\widehat{\mathbb{S}}_{2}, \widehat{\mathbb{S}}_{1}\right]_{1}, \widehat{\mathbb{X}}_{2}\right]_{2}} \\
& -i \frac{61}{450} \sqrt{\frac{1}{14}}\left[\left[\widehat{\mathscr{A}}_{2}, \widehat{S}_{1}\right]_{3}, \widehat{\mathscr{S}}_{1}\right]_{2}+i \frac{287}{180} \sqrt{\frac{1}{10}}\left[\left[\widehat{\mathbb{X}}_{2}, \widehat{S}_{1}\right]_{2}, \widehat{S}_{1}\right]_{2}+i \frac{13}{100} \sqrt{\frac{1}{6}}\left[\left[\widehat{\mathbb{X}}_{2}, \widehat{S}_{1}\right]_{1}, \widehat{S}_{1}\right]_{2} \\
& +\frac{116}{25} \sqrt{\frac{2}{7}}\left\{\widehat{X}_{1},\left[\widehat{S}_{2}, \widehat{S}_{1}\right]_{3}\right\}_{2}-\frac{68}{15} \sqrt{\frac{2}{5}}\left\{\widehat{X}_{1},\left[\widehat{S}_{2}, \widehat{S}_{1}\right]_{2}\right\}_{2}+\frac{51}{25} \sqrt{\frac{3}{2}}\left\{\widehat{X}_{1},\left[\widehat{S}_{2}, \widehat{S}_{1}\right]_{1}\right\}_{2} \\
& -\frac{i}{2} \sqrt{\frac{1}{5}}\left\{\widehat{X}_{1},\left[\widehat{S}_{1}, \widehat{S}_{1}\right]_{1}\right\}_{2}-i \frac{436}{5} \sqrt{\frac{2}{105}}\left(\widehat{\mathbb{X}}_{2}^{2}\right)_{2}-i \frac{171}{5} \sqrt{\frac{6}{35}}\left(\widehat{S}_{2}^{2}\right)_{2} \\
& -\frac{1}{2} \sqrt{\frac{1}{15}}\left\{\widehat{S}_{2}, \widehat{S}_{1}\right\}_{2}-i \frac{4412}{15} \sqrt{\frac{2}{105}}\left\{\left(\widehat{X}_{1}^{2}\right)_{2}, \widehat{X}_{2}\right\}_{2}+i \frac{616}{15} \sqrt{\frac{2}{15}}\left\{\left(\widehat{X}_{1}^{2}\right)_{0}, \widehat{X}_{2}\right\}_{2} \\
& -i \frac{1152}{35} \sqrt{\frac{6}{5}}\left\{\left(\widehat{X}_{1}^{2}\right)_{0},\left(\widehat{X}_{1}^{2}\right)_{2}\right\}_{2}+i\left(\frac{10492}{75}-\frac{109}{10} f\right) \sqrt{\frac{1}{10}} \widehat{\mathbb{X}}_{2} \\
& -i\left(\frac{39168}{175}+\frac{78}{5} f\right) \sqrt{\frac{2}{5}}\left(\widehat{X}_{1}^{2}\right)_{2} \text {; } \\
& {\left[\left[\widehat{\mathbb{S}}_{2}, \widehat{\mathbb{S}}_{1}\right]_{3}, \widehat{\mathbb{X}}_{2}\right]_{2}-\sqrt{\frac{2}{5}}\left[\left[\widehat{\mathbb{S}}_{2}, \widehat{\mathscr{S}}_{1}\right]_{2}, \widehat{\mathbb{X}}_{2}\right]_{2}+\frac{1}{3} \sqrt{14}\left[\left[\widehat{\mathbb{S}}_{2}, \widehat{\mathbb{S}}_{1}\right]_{1}, \widehat{\mathbb{X}}_{2}\right]_{2}-i \frac{44}{45}\left[\left[\widehat{\mathbb{X}}_{2}, \widehat{\mathbb{S}}_{1}\right]_{3}, \widehat{\mathbb{S}}_{1}\right]_{2}} \\
& -\frac{i}{9} \sqrt{\frac{7}{5}}\left[\left[\widehat{\mathbb{X}}_{2}, \widehat{S}_{1}\right]_{2}, \widehat{S}_{1}\right]_{2}+\frac{i}{5} \sqrt{\frac{7}{3}}\left[\left[\widehat{\mathbb{X}}_{2}, \widehat{S}_{1}\right]_{1}, \widehat{S}_{1}\right]_{2}-\frac{116}{45}\left\{\widehat{\mathbb{X}}_{1},\left[\widehat{S}_{2}, \widehat{S}_{1}\right]_{3}\right\}_{2} \\
& +\frac{16}{9} \sqrt{\frac{7}{5}}\left\{\widehat{X}_{1},\left[\widehat{S}_{2}, \widehat{S}_{1}\right]_{2}\right\}_{2}+\frac{4}{5} \sqrt{\frac{7}{3}}\left\{\widehat{\mathbb{X}}_{1},\left[\widehat{\mathscr{S}}_{2}, \widehat{S}_{1}\right]_{1}\right\}_{2}+i 64 \sqrt{\frac{1}{15}}\left(\widehat{\mathbb{X}}_{2}^{2}\right)_{2} \\
& +i 24 \sqrt{\frac{3}{5}}\left(\widehat{S}_{2}^{2}\right)_{2}-\frac{2}{3} \sqrt{\frac{70}{3}}\left\{\widehat{S}_{2}, \widehat{S}_{1}\right\}_{2}+i \frac{608}{3} \sqrt{\frac{1}{15}}\left\{\left(\widehat{X}_{1}^{2}\right)_{2}, \widehat{\mathbb{X}}_{2}\right\}_{2} \\
& -i \frac{64}{3} \sqrt{\frac{7}{15}}\left\{\left(\widehat{X}_{1}^{2}\right)_{0}, \widehat{X}_{2}\right\}_{2}+i 128 \sqrt{\frac{3}{35}}\left\{\left(\widehat{X}_{1}^{2}\right)_{0},\left(\widehat{X}_{1}^{2}\right)_{2}\right\}_{2}-i\left(\frac{704}{15}-4 f\right) \sqrt{\frac{7}{5}} \widehat{X}_{2} \\
& +i\left(\frac{5472}{5}+84 f\right) \sqrt{\frac{1}{35}}\left(\widehat{X}_{1}^{2}\right)_{2} \text {; }
\end{aligned}
$$




$$
\begin{aligned}
& J^{P}=2^{-} \text {: } \\
& {\left[\left[\widehat{\mathbb{S}}_{2}, \widehat{\mathbb{S}}_{1}\right]_{1}, \widehat{\mathbb{S}}_{2}\right]_{2}-i \frac{11}{15} \sqrt{\frac{7}{2}}\left[\left[\widehat{\mathbb{S}}_{2}, \widehat{\mathbb{S}}_{1}\right]_{3}, \widehat{\mathbb{S}}_{1}\right]_{2}+i \frac{7}{6} \sqrt{\frac{1}{10}}\left[\left[\widehat{\mathbb{S}}_{2}, \widehat{\mathbb{S}}_{1}\right]_{2}, \widehat{\mathbb{S}}_{1}\right]_{2}} \\
& +\frac{i}{10} \sqrt{\frac{3}{2}}\left[\left[\widehat{\mathscr{S}}_{2}, \widehat{S}_{1}\right]_{1}, \widehat{S}_{1}\right]_{2}-i \frac{3}{5}\left\{\widehat{X}_{1},\left[\widehat{\mathscr{X}}_{2}, \widehat{S}_{2}\right]_{1}\right\}_{2}+\frac{1}{5} \sqrt{\frac{7}{2}}\left\{\widehat{X}_{1},\left[\widehat{\mathbb{X}}_{2}, \widehat{S}_{1}\right]_{3}\right\}_{2} \\
& -\frac{7}{3} \sqrt{\frac{1}{10}}\left\{\widehat{X}_{1},\left[\widehat{\mathbb{X}}_{2}, \widehat{S}_{1}\right]_{2}\right\}_{2}-\frac{4}{5} \sqrt{6}\left\{\widehat{\mathbb{X}}_{1},\left[\widehat{\mathbb{X}}_{2}, \widehat{S}_{1}\right]_{1}\right\}_{2}+i \sqrt{\frac{42}{5}}\left\{\widehat{\mathbb{X}}_{2}, \widehat{S}_{2}\right\}_{2} \\
& -i \frac{172}{15} \sqrt{\frac{14}{15}}\left\{\left(\widehat{X}_{1}^{2}\right)_{2}, \widehat{S}_{2}\right\}_{2}+i \frac{56}{15} \sqrt{\frac{2}{15}}\left\{\left(\widehat{X}_{1}^{2}\right)_{0}, \widehat{S}_{2}\right\}_{2}+48 \sqrt{\frac{3}{5}}\left\{\left(\widehat{X}_{1}^{2}\right)_{2}, \widehat{S}_{1}\right\}_{2} \\
& +i\left(\frac{92}{3}-\frac{21}{2} f\right) \sqrt{\frac{1}{10}} \widehat{\mathbb{S}}_{2} \text {; } \\
& {\left[\left[\widehat{\mathscr{S}}_{1}, \widehat{S}_{1}\right]_{1}, \widehat{\mathscr{S}}_{1}\right]_{2}+i 24 \sqrt{\frac{1}{5}}\left\{\widehat{\mathbb{X}}_{1},\left[\widehat{\mathbb{X}}_{2}, \widehat{\mathbb{S}}_{2}\right]_{1}\right\}_{2}-2 \sqrt{\frac{14}{5}}\left\{\widehat{X}_{1},\left[\widehat{\mathbb{X}}_{2}, \widehat{S}_{1}\right]_{3}\right\}_{2}} \\
& +2 \sqrt{2}\left\{\widehat{X}_{1},\left[\widehat{\mathbb{X}}_{2}, \widehat{\mathbb{S}}_{1}\right]_{2}\right\}_{2}-6 \sqrt{\frac{6}{5}}\left\{\widehat{X}_{1},\left[\widehat{\mathbb{X}}_{2}, \widehat{S}_{1}\right]_{1}\right\}_{2}-4 \sqrt{3}\left\{\widehat{\mathbb{X}}_{2}, \widehat{\mathbb{S}}_{1}\right\}_{2} \\
& +i \frac{32}{5} \sqrt{\frac{14}{3}}\left\{\left(\widehat{X}_{1}^{2}\right)_{2}, \widehat{S}_{2}\right\}_{2}+i \frac{224}{5} \sqrt{\frac{2}{3}}\left\{\left(\widehat{X}_{1}^{2}\right)_{0}, \widehat{S}_{2}\right\}_{2}+40 \sqrt{3}\left\{\left(\widehat{X}_{1}^{2}\right)_{2}, \widehat{S}_{1}\right\}_{2}-i \frac{16}{5} \sqrt{2} \widehat{\mathscr{S}}_{2} \text {; } \\
& J^{P}=1^{+} \text {: } \\
& {\left[\left[\widehat{\mathbb{S}}_{2}, \widehat{\mathbb{S}}_{1}\right]_{3}, \widehat{\mathbb{X}}_{2}\right]_{1}-\frac{1}{2} \sqrt{35}\left[\left[\widehat{\mathbb{S}}_{2}, \widehat{\mathbb{S}}_{1}\right]_{2}, \widehat{\mathbb{X}}_{2}\right]_{1}+\frac{1}{2} \sqrt{21}\left[\left[\widehat{\mathbb{S}}_{2}, \widehat{\mathbb{S}}_{1}\right]_{1}, \widehat{\mathbb{X}}_{2}\right]_{1}} \\
& -\frac{i}{2} \sqrt{\frac{105}{2}}\left[\left[\widehat{\mathbb{X}}_{2}, \widehat{\mathscr{S}}_{1}\right]_{2}, \widehat{\mathbb{S}}_{1}\right]_{1}+\frac{i}{2} \sqrt{\frac{35}{2}}\left[\left[\widehat{\mathbb{X}}_{2}, \widehat{\mathscr{S}}_{1}\right]_{1}, \widehat{\mathbb{S}}_{1}\right]_{1}+\sqrt{210}\left\{\widehat{\mathbb{X}}_{1},\left[\widehat{\mathscr{S}}_{2}, \widehat{\mathbb{S}}_{1}\right]_{2}\right\}_{1} \\
& +3 \sqrt{35}\left\{\widehat{S}_{2}, \widehat{S}_{1}\right\}_{1}+i 8 \sqrt{210}\left\{\left(\widehat{X}_{1}^{2}\right)_{2}, \widehat{X}_{2}\right\}_{1} ; \\
& J^{P}=1^{-} \text {: } \\
& {\left[\left[\widehat{\mathscr{S}}_{2}, \widehat{\mathbb{S}}_{1}\right]_{1}, \widehat{\mathscr{S}}_{2}\right]_{1}-i \frac{11}{4} \sqrt{\frac{1}{10}}\left[\left[\widehat{\mathbb{S}}_{2}, \widehat{\mathscr{S}}_{1}\right]_{2}, \widehat{\mathscr{S}}_{1}\right]_{1}+i \frac{5}{4} \sqrt{\frac{5}{6}}\left[\left[\widehat{\mathbb{S}}_{2}, \widehat{\mathscr{S}}_{1}\right]_{1}, \widehat{\mathscr{S}}_{1}\right]_{1}} \\
& +\frac{7}{24}\left[\left[\widehat{\mathscr{S}}_{1}, \widehat{S}_{1}\right]_{1}, \widehat{S}_{1}\right]_{1}-i \frac{3}{2} \sqrt{\frac{1}{5}}\left\{\widehat{X}_{1},\left[\widehat{\mathbb{X}}_{2}, \widehat{S}_{2}\right]_{1}\right\}_{1}-i 6 \sqrt{\frac{1}{5}}\left\{\widehat{X}_{1},\left[\widehat{\mathbb{X}}_{2}, \widehat{S}_{2}\right]_{0}\right\}_{1} \\
& -\frac{7}{3} \sqrt{10}\left\{\widehat{X}_{1},\left[\widehat{\mathbb{X}}_{2}, \widehat{\mathscr{S}}_{1}\right]_{2}\right\}_{1}+\frac{49}{2} \sqrt{\frac{1}{30}}\left\{\widehat{X}_{1},\left[\widehat{\mathbb{X}}_{2}, \widehat{\mathscr{S}}_{1}\right]_{1}\right\}_{1}+i 3 \sqrt{\frac{1}{10}}\left\{\widehat{\mathbb{X}}_{2}, \widehat{\mathscr{S}}_{2}\right\}_{1} \\
& -\frac{49}{2} \sqrt{\frac{1}{15}}\left\{\widehat{X}_{2}, \widehat{S}_{1}\right\}_{1}+i \frac{32}{3} \sqrt{10}\left\{\left(\widehat{X}_{1}^{2}\right)_{2}, \widehat{S}_{2}\right\}_{1}-\frac{161}{3} \sqrt{\frac{1}{15}}\left\{\left(\widehat{X}_{1}^{2}\right)_{2}, \widehat{S}_{1}\right\}_{1} \\
& +\frac{98}{3} \sqrt{\frac{1}{3}}\left\{\left(\widehat{X}_{1}^{2}\right)_{0}, \widehat{\mathscr{S}}_{1}\right\}_{1}+\left(\frac{98}{15}-\frac{49}{8} f\right) \widehat{\mathbb{S}}_{1} \text {. }
\end{aligned}
$$

\section{Quantum actions of $\widehat{\mathcal{B}}_{0}^{(1)}$ :}

$$
\begin{aligned}
& {\left[\widehat{\mathcal{B}}_{0}^{(1)}, \widehat{\mathbb{X}}_{2}\right]_{2}=i \sqrt{6}\left[\widehat{\mathfrak{S}}_{2}, \widehat{\mathcal{S}}_{1}\right]_{2}} \\
& {\left[\widehat{\mathcal{B}}_{0}^{(1)}, \widehat{\mathfrak{S}}_{2}\right]_{2}=-i 2 \sqrt{\frac{2}{3}}\left[\widehat{\mathbb{X}}_{2}, \widehat{\mathfrak{S}}_{1}\right]_{2}+2 \sqrt{\frac{2}{3}}\left\{\widehat{\mathbb{X}}_{1}, \widehat{\mathfrak{S}}_{2}\right\}_{2}+i 6\left\{\widehat{\mathscr{X}}_{1}, \widehat{\mathcal{S}}_{1}\right\}_{2} ;} \\
& {\left[\widehat{\mathcal{B}}_{0}^{(1)}, \widehat{\mathcal{S}}_{1}\right]_{1}=-i 6 \sqrt{\frac{2}{5}}\left[\widehat{\mathbb{K}}_{2}, \widehat{\mathcal{S}}_{2}\right]_{1}+2 \sqrt{\frac{3}{5}}\left[\widehat{\mathbb{X}}_{2}, \widehat{\mathcal{S}}_{1}\right]_{1}-i 12 \sqrt{\frac{3}{5}}\left\{\widehat{\mathscr{X}}_{1}, \widehat{\mathcal{S}}_{2}\right\}_{1}-6 \sqrt{2}\left\{\widehat{\mathscr{X}}_{1}, \widehat{\mathcal{S}}_{1}\right\}_{1} \text {. }}
\end{aligned}
$$

Remarks:

1.: Each restriction on the deformation parameters originates from one or more consistency relations with degree $(l+2)$ in the form of a condition stating that the coefficient in front of a given selected basis element of a certain subspace $\widehat{\mathfrak{F}}_{j, \pm}^{l^{\prime}}, l^{\prime} \leq l+1$, must vanish. This allows to assign a definite order in Planck's constant, viz. the order $\left(l+2-l^{\prime}\right) \geq 1$, to each equation from a complete collection of parameter restrictions 
when the latter are presented in their original form.

2.: The common numerical value zero determined above for all twenty-six parameters, initially of first order in Planck's constant, and of one parameter, initially of third order in Planck's constant, does not come as a surprise. Indeed, notice that the inhomogeneities of the system of equations for the parameters arise exclusively from rearrangements of double anticommutators or, more specifically, from the contributions of the respective double commutators to these rearrangements. Thus, provided the parameter restrictions are presented in their original form, the inhomogeneities appear only in parameter equations of even positive integer order in Planck's constant and, in particular, not in the equations of first and third order determining the aforementioned $26+1$ parameters.

3.: Rescaling, if necessary, the deformation parameters by square roots of appropriate rational numbers, it is possible to give all independent parameter equations derived in the course of the cycles of degree $\leq(l+1)$ simultaneously a polynomial form with rational coefficients. Moreover, without loss of generality, it may be assumed that a definite order in Planck's constant is assigned to each parameter equation in such form.

Based on the experience gained by the cycles of degree two and three, I shall risk a prognosis for the next cycle $(l+1)=4$ :

1.: There is a good chance that the one residual free parameter $f$ will be fixed at a rational value in the course of the next cycle $($ i.e. $l+1=4)$ by the consistency requirements for all $\widehat{\mathfrak{U}}$-relations with degree 5 , spin and parity $J^{P}=4^{+}$induced from $\widehat{\mathfrak{U}}$-relations with degrees $\leq 4$. Typically, each such non-trivial consistency condition would give rise to 6 linear inhomogeneous equations for the only indeterminate $f$. The consistency of all $\widehat{\mathfrak{U}}$-relations with degree 5 , spin and parity $J^{P}=6^{ \pm}, 5^{ \pm}$obtained by induction from the $\widehat{\mathfrak{U}}$-relations (and the $\widehat{\mathcal{B}}_{0}^{(l)}$-actions) with degree $\leq 4$ is already guaranteed by the consistency of the classical algebra $\mathfrak{h}$ : on the one hand the quantum corrections for the $\widehat{\mathfrak{U}}$-generating relations with degree $\leq 4$ do not survive these inductions, and on the other hand rearrangements of the various terms carrying spin and parity $J^{P}=6^{ \pm}, 5^{ \pm}$cannot produce inhomogeneities since the subspaces $\widehat{\mathfrak{U}}_{6, \pm}^{3}, \widehat{\mathfrak{U}}_{5, \pm}^{3}$ consist of the zero element only.

2.: In the course of this next cycle $(l+1=4)$, the classical action of $\mathcal{B}_{0}^{(3)}$ on the generators of $\mathfrak{U}$ is deformed into the quantum action of $\widehat{\mathcal{B}}_{0}^{(3)}$ at the expense of $47+9$ +4 real parameters, in their initial form of first, second and third order in Planck's constant, respectively.

At first sight this rapidly growing number of free parameters may cause a shock. However, a closer look suggests that the number of consistency restrictions on these parameters grows even more rapidly. As a matter of fact, $\widehat{\mathcal{B}}_{0}^{(3)}$ contributes to the $\widehat{\mathfrak{U}}$-relations with degree $l+2=5$ in two ways: in the first place it promotes the $\widehat{\mathfrak{U}}-$ relations with degree 2 to $\widehat{\mathfrak{U}}$-relations with degree 5 (one $J^{P}=4^{-}-$, two $J^{P}=3^{+-}$, 
one $J^{P}=3^{-}$, one $J^{P}=2^{-}$and one $J^{P}=1^{+}$-relation) and in the second place, by the postulated commutativity of $\widehat{\mathcal{B}}_{0}^{(1)}$ and $\widehat{\mathcal{B}}_{0}^{(3)}$, it provides three more $\widehat{\mathfrak{U}}$-relations with degree 5 (for $J^{P}=2^{+}, 2^{-}, 1^{-}$one relation each). But then, consistency requires among other things that the commutator action of $\widehat{\mathcal{B}}_{0}^{(3)}$ annihilates the ele-

ment $\left[\widehat{\mathbb{X}}_{2}, \widehat{S}_{2}\right]_{4}$. This condition by itself, confronted with the rest of the $\widehat{\mathfrak{U}}$-relations with degree 5 , spin and parity $4^{-}$, will already furnish 19 linear homogeneous and 4 quadratic inhomogenous equations for the 38 real parameters involved: 32 of them initially of order $\hbar^{1}$, and the remaining 6 parameters (including the residual parameter $f$ ) initially of order $\hbar^{2}$.

3.: It is obvious from the order of $\widehat{\mathcal{B}}_{0}^{(3)}$ in Planck's constant that the 60 real indeterminates parametrizing the quantum action of $\widehat{\mathcal{B}}_{0}^{(3)}$ appear only linearly in the parameter equations which turn up as consistency conditions in the course of the deformation cycle of degree $(l+1)=4$. If one way or another the numerical value of the parameter $f$ can be determined as a rational number, the system of equations for the remaining parameters will decouple into two subsystems: one subsystem consisting of the homogeneous linear equations with rational coefficients of first and third (possibly even fifth) order in Planck's constant for the $47+4$ parameters, initially of first and third order in Planck's constant, the other subsystem consisting of the inhomogeneous linear equations with rational coefficients of second and fourth order in Planck's constant for the 9 parameters, initially of second order in Planck's constant. If the equations of the first subsystem combine to determine some or all parameters involved, the values of these parameters must be zero. If the analogous assumption applies to the second subsystem, the values of the pertinent parameters will be rational numbers. It is tempting to speculate that ultimately all parameters will be determined, those involved in the first subsystem necessarily as zero, those involved in the second subsystem necessarily as rational numbers.

If the speculation is borne out by facts, then the vanishing of the former parameters would suggest that in the quantum theory a $\mathbb{Z}_{2}$-grading survives as a reminiscence of the classical $\mathbb{N}_{0}$-grading w.r.t. the degree $l$, whereas the rational values of the latter parameters would match well with the circumstance that the classical invariant charges form a Poisson algebra with integer numbers as "structure constants".

\section{Conclusions}

Exact quantum theoretic information in such detail as above is available only for systems with an exceptionally large symmetry group. In the context of physics the interest in such a system is clearly justified if this system serves as a model for some concrete physical phenomenon providing a satisfactory description of the principal features of the phenomenon. Apart from that, the interest in such a system is also justified if as a model this system by itself leaves much to be desired, but if - as far as mathematical structure, concepts and methods are concerned - this system serves 
as a good starting point for a systematic improvement and extension procedure. As I see it, the interest in the Nambu-Goto theory and in its structural analysis is of the second kind.

On account of the rapidly growing computational demands, alternative ways of gathering information must be explored in order to arrive at a purely algebraic description of the most important observable features of the Nambu-Goto theory. One such way is the clarification of the bialgebra aspects of the quantum algebra of observables. The non-additive composition laws for the invariant charges of two branches of a string trajectory merging in a single branch, which have been established in Ref. [7], hint at the existence of a non-trivial coproduct of the algebra of observables. The adequate setting for the construction of such a coproduct is the minimal algebra embedding the individual algebras corresponding to the three separate branches. This embedding algebra contains also elements which cannot be affiliated with any branch as piecewise conserved charges, which must therefore be assigned to the vertex itself.

Also for another reason this algebra is interesting: the energy-momentum operators corresponding to the individual merging branches cease to be central elements, and so do their mass squares. This means that, apart from the operators which were studied so far and which raise the degree, the algebra contains also operators lowering the degree. With their help additional diagonalizable elements with corresponding spectra/roots can be produced beyond those which furnish the quantum labels degree, spin and parity employed throughout.

It was pointed out to me by L. Tisza that the idea to formulate the observable features of a continuum field theory in purely algebraic terms and their physical interpretation was already pondered by A. Einstein [2]. This fact does not seem to be a matter of common knowledge.

Acknowledgements: I thank G. Handrich for clarifying discussions and for taking his share in the tedious calculations. Computational assistance by him as well as by T. Fischer, especially in the form of a final computer check of the relations, is gratefully acknowledged.

I would like to thank the staff of the Physics Department of the University of California at Berkeley for the kind hospitality extended to me during the fall of 1996 and the spring of 1997.

This work was supported in part by Volkswagen-Stiftung and in part by U.S. National Science Foundation under grant PHY-95-14797. 


\section{References}

[1] Brink, L., Henneaux, M.: Principles of String Theory. New York, London: Plenum Press 1988

[2] Einstein, A.: The Meaning of Relativity. 5th edition, 4th Princeton Paperback Printing, p. 165. Princeton: Princeton University Press 1974

[3] Gerdt, V.P., Kornyak, V.V.: Construction of Finitely Presented Lie Algebras and Superalgebras. J. Symb. Comput. 21, 337 (1996)

[4] Rotenberg, M., Bivins, R., Metropolis, N., Wooten, J.K. (Jr.): The 3-j and 6-j Symbols. Cambridge, Massachusetts: The Technology Press, Massachusetts Institute of Technology 1959

[5] Niederer, U.H., O'Raifeartaigh, L.: Realizations of the Unitary Representations of the Inhomogeneous Space-Time Groups. Fortschritte der Physik 22, $111(1974)$

[6] Pohlmeyer, K.: Uncovering the Detailed Structure of the Algebra Formed by the Invariant Charges of Closed Bosonic Strings Moving in 1+2-Dimensional Minkowski Space. Commun. Math. Phys. 163, 629 (1994)

[7] Pohlmeyer, K.: The Invariant Charges of the Nambu-Goto Theory: NonAdditive Composition Laws. Mod. Phys. Lett. 10, 295 (1995)

[8] Pohlmeyer, K., Rehren, K.-H.: Algebraic Properties of the Invariant Charges of the Nambu-Goto Theory. Commun. Math. Phys. 105, 593 (1986)

[9] Pohlmeyer, K., Rehren, K.-H.: The Algebra Formed by the Invariant Charges of the Nambu-Goto Theory: Identification of a Maximal Abelian Subalgebra. Commun. Math. Phys. 114, 55 (1988)

[10] Stanley, R.P.: Enumerative Combinatorics. Vol I, ch. 3 and 4. The Wadsworth \& Brooks/Cole Mathematics Series. Monterey, California: Wadsworth \& Brooks/Cole 1986 